\title{
WestVirginiaUniversity
}

THE RESEARCH REPOSITORY @ WVU

West Virginia Agricultural and Forestry Experiment

Davis College of Agriculture, Natural Resources

Station Bulletins

And Design

$1-1-1908$

\section{Commercial Fertilizers : Complete Report for 1908}

\author{
J. H. Stewart
}

B. H. Hite

Follow this and additional works at: https://researchrepository.wvu.edu/ wv_agricultural_and_forestry_experiment_station_bulletins

\section{Digital Commons Citation}

Stewart, J. H. and Hite, B. H., "Commercial Fertilizers : Complete Report for 1908" (1908). West Virginia Agricultural and Forestry Experiment Station Bulletins. 118.

https://researchrepository.wvu.edu/wv_agricultural_and_forestry_experiment_station_bulletins/118 @ WVU. It has been accepted for inclusion in West Virginia Agricultural and Forestry Experiment Station Bulletins by an authorized administrator of The Research Repository @WVU. For more information, please contact ian.harmon@mail.wvu.edu. 



\title{
WEST VIRGINIA UNIVERSITY \\ AGRICULTURAL EXPERIMENT STATION \\ MORGANTOWN, W. VA.
}

BULLE'TIN II 8

DECEMBER 3I, I9O8

\section{Commercial Fertilizers}

\section{COMPLETE REPORT}

\author{
FOR \\ 1908
}

By J. H. STEWART AND B. H. HITE

[The Bulletins and Reports of this Station will bo mailed free to any citizen of West Virginia upon written application. Address: Director of Agricultural Experiment Station, Morgantown, W. Va.]: 


\section{REGENTS OF THE WEST VIRGINIA UNIVERSITY}

Name of Regent

P. O. Address

Hon. C. M. Babi..................Falls, W. Va. Hon. J. B. FinLEY. . . . . . . . . . Parkersburg, W. Va. Hon. D. C. Galla AER ............... Charleston, W. Va . Hon. E. M. Grant................... Hon. C. E. HaworTH............. Huntington, W. Va. Ion. C. P. MCNell. .............Wheeling, W. Va. Hon. L. J. Wirlians. .............. Lewisburg, W. Va. Hon. T. P. JAcobs............... New Martinsville, W. Va. HoN. I. R. TROTTER..................

President of the Board of Regents .......... . M. BABB President of the University............. B. Purinton Treasurer...................... R. WhiteHILL Auditor....................... White

\section{STATION STAFF}

TAMES H. STEWART, A. M......... Director and Agriculturist BERT H. HitE, M. S............Vice Director and Chemist W. M. Munson, PH. D................. Horticulturist W. E. Rumsey. B. S. Agr. . . . . . . . . . . Entomologist

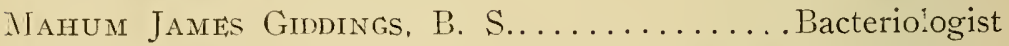
Horace Atwood, M. S. Agr............ Assistant Agricu'turist Fren E. Brooks ................. Associate Entomologist Frank B. Kunst, A. B................ Assistant Chemist Chas. E. WEAKLEY, JR...............Assistant Chemist

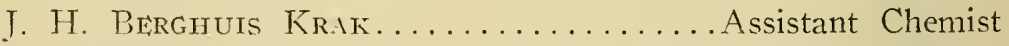

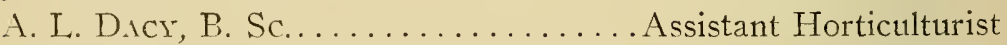

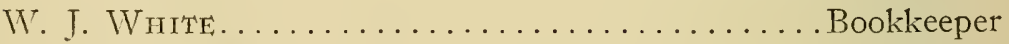

M. A. STEWART . . . . . . . . . . . . . . . Librarian ALICE ENGLE. . . . . . . . . . . . . . . . . Stenographer B. D. WHITE. . . . . . . . . . . . . . . . . . Herdsman Theо. F. Iмвıсн..................... Greenhouseman 


\section{Commercial Fertilizers}

Complete Report for 1908

Bulletin 118

The following is a complete report of the work required of this Experiment Station by the Fertilizer Law of this State during the year ending Dec. 3I, I908.

This report shows that there is an abundance of excellent fertilizers for all purposes regularly on sale in almost every section of the State. There is also an abundance of low grade fertilizers and a very manifest determination to dump as much of them as possible into this State. This involves no violation of any statute. When the manufacturers of these low grade fertilizers state that they are low grade, make oath to the statement and pin it all over the packages, there is nothing more that legislation can do.

The losses and disappointments attending the use of low grade fertilizers were treated at some length in the Fertilizer Report of last year, copies of which may yet be had for the asking.

THE AMERICAN AGRICULTURAL CHEMICAL CO..

No. 2 Rector Strect, New York City' and $74 I$ Equitable Building, Baltimore, $M d$.

6043. A. A. C. Co.'s Pure Ground Bone, Thos. Nuzum, Agent, Grafton, W. Va. Guarantee: Phosphoric acid, total, 20.60 ; nitrogen, 3.30 ; phosphoric acid from animal bone, nitrogen from animal bone. Found: Phosphoric acid, total, 24.54: nitrogen, 3.53; availability of nitrogen, 89. 
6187. A. A. C. Co.'s Pure Ground Bone, Thos. Nuzum. Agent, Grafton, W. Va. Guarantee: (As above). Found: Phosphoric acid, total, 23.64; nitrogen, 3.76; availability of nitrogen, 85 .

6050. A. A. C. Co.'s Fine Ground Bone, Johnson \& Gwinn, Agent, Alderson, W. Va. Guarantee: Phosphoric acid, total, 22.80; nitrogen, 2.47; phosphoric acid from animal bone, nitrogen from animal bone. Found: Phosphoric acid, total, 23.99; nitrogen, 2.38; availability of nitrogen, 93.

6096. A. A. C. Co.'s Bone Meal, Siever Hardware Co.. Agent, Keyser, W. Va. Guarantee: Phosphoric acid, total, r 3.75 ; nitrogen, 1.65; phosphoric acid from animal bone, nitrogen from animal bone. Fonnd: Phosphoric acid, total, I5.19; nitrogen, I.6I ; availability of nitrogen, 89 .

6042. A. A. C. Co.'s Gem Alkaline Phosphate, Thos. Nuzum, Agent, Grafton, W. Va. Guarantee: Phosphoric acid, soluble, 4; reverted, 2 ; insoluble, 1 ; total, 7 ; available, 6; potash, 3; phosphoric acid from dissolved phosphate rock; potash from kainit. Found: Phosphoric acid, soluble, 2.32; reverted, 3.91 ; insoluble, 0.40 ; total, 6.63 ; available, 6.23 ; potash, 2.90 ; chlorine, 6.2.

Remark: Chlorine excessive.

6084. A. A. C. Co.'s Gem Alkaline Phosphate, T. B. Drummond \& Co., Agent, Buckhannon, W. Va. Guarantee: (As above). Found: Phosphoric acid, soluble, 1.80; reverted. 4.52 ; insoluble, 0.38 ; total, 6.70; available, 6.32; potash, 3.20 ; chlorine, $5 \cdot 5$.

Remark: Chlorine excessive.

6r32. A. A. C. Co.'s Gem Alkaline Phosphate, R. Hunter, Agent, Berkeley Springs, W. Va. Guarantee: (As above). Found: Phosphoric acid, soluble, 2.25; reverted, 4.05; insoluble, 0.40 ; total, 6.70; available, 6.30; potash, 3.63; chlorine, 4.8 .

Remark: Chlorine excessive. 
6049. Big Crop Phospliate, Johnson \& Gwinn, Alderson, W. Va. Guarantee: Phosphoric acid, soluble, 6; reverted, 2; insoluble, I ; total, 9; available, 8; potash, 5; phosphoric acid from dissolved phosphate rock, potash from manure salt. Found: Phosphoric acid, soluble, 4.I8; reverted, 4.05: insoluble, 0.55 ; total, 8.78 ; available, 8.23 ; potash, 5.06 ; chlorine; 2.8 .

Remark: Source of potash equivalent to kainit.

608I. Big Crop Phosphate, T. B. Drummond \& Co., Agent, Buckhannon, W. Va. Guarantee: (As above). Found: Phosphoric acid, soluble, 3.Io; reverted, 5.4I, insoluble, 0.70: total, 9.2I ; available, 8.5I ; potash, 5.36; chlorine, 3 .

Remark: Source of potash equivalent to kainit.

6206. Dissolved Animal Bone, T. P. Licklider, Agent, Martinsburg, W. Va. Guarantee: Phosphoric acid, soluble, Io; reverted, 2 ; insoluble, I ; total, I3 ; available, I2 ; nitrogen, 2.06; phosphoric acid from animal bone, 'nitrogen from animal bone. Found: Phosphoric acid, soluble, 9.54; reverted, 2.62 ; insoluble, 3.I9; total, I5.35; available, I2.I6; nitrogen, 2.27 ; availability of nitrogen, 92 .

6022. Corn, Oats and Buckwheat Fertilizer, Cicero Phillips, Agent, Belington, W. Va. Guarantee: Phosphoric acid. soluble, 4; reverted, 2; insoluble, I ; tota1, 7; available, 6; potash, 3; phosphoric acid from dissolved phosphate rock, potash from kainit. Found: Phosphoric acid, soluble, 2.or ; reverted, 4.56 ; insoluble, 0.47 ; total, 7.04 ; available, 6.57 ; potash, 2.90 ; chlorine, 5.8 .

Remark: Chlorine excessive.

6040. High Grade Dissolved Bone \& Potash, Thos. Nuzum, Agent, Grafton, W. Va. Guarantee: Phosphoric acid, soluble, 8; reverted, 2 ; insoluble, I; total, II ; available, Io ; potash, 5; phosphoric acid from dissolved phosphate rock, potash $I / 4$ to $3 / 4$ manure salts, $I / 4$ to $3 / 4$ muriate of potash. 
Found: Phosphoric acid, soluble, 5.58; reverted, 4.44; insoluble, 0.56; total, I0.58; available, I0.02; potash, 5.76 : chlorine, 3 .

Remark: Source of potash equivalent to kainit.

6127. Genuine German Kainit, Washington, Alexander \& Cooke, Charlestown, W. Va. Guarantee: Potash, I2: potash from kainit. Found: Potash, I2.06; chlorine, 2.8.

6203. Super-phosphate, Geo. B. Hooff, Charlestown, W. Va. Guarantee: Phosphoric acid, soluble, I4; reverted, 2: insoluble, I; total, I7; available, I6: phosphoric acid from dissolved phosphate rock. Found: Phosphoric acid, soluble. 8.62; reverted, 9.32; insoluble, 0.24; total, I8.I8; available. I7.94.

604I. Bradley's Bean \& Potato Phosphate, Thos. Nuzum, Agent, Grafton, W. Va. Guarantee: Phosphoric acid. soluble, 6; reverted, 2; insoluble, I; total, 9; available, 8; nitrogen, 0.82 ; potash, 4 ; phosphoric acid from dissolved phosphate rock and dissolved animal bone, nitrogen from animal tankage, potash $\mathrm{T} / 4$ to $3 / 4$ from manure salts, $1 / 4$ to $3 / 4$ kainit. Found: Phosphoric acid, soluble, 4.23; reverted, 4.22: in. soluble, I.I8; total, 9.63; available, 8.45 ; nitrogen, .98: potash. 4.48: availability of nitrogen, 77 ; chlorine, 2.7 .

Remark: Chlorine excessive.

6I76. Bradley's Bean \& Potato Phosphate, Thompson \& Ireland, Worthington, W. Va. Guarantee: (As above). Found: Phosphoric acid, soluble, 4.22; reverted, 4.33; insoluble, I.39: total, 9.94 ; available, 8.55 ; nitrogen, 1.07 ; potash, 4.63 : availability of nitrogen, 77 ; chlorine, 2.5 .

6044. Bradley's Niagara Phosphate, Thos. Nuzum. Agent, Grafton, W. Va. Guarantee: Phosphoric acid, soluble. 5: reverted, 2 ; insoluble, I ; total, 8; available, 7 ; nitrogen. 0.82 ; potash, I; phosphoric acid from dissolved animal bone 
and dissolved phosphate rock, nitrogen from animal tankage, potash from kainit. Found: Phosphoric acid, soluble, 3.52; reverted, 3.72 ; insoluble, 0.92 ; total, 8.16 ; available, 7.24 ; nitrogen, 0.97 ; potash, I.2I ; availability of nitrogen, 78 ; chlorine, 6.7 .

Remark: Chlorine excessive.

608o. Bradley's Niagara Phosphate, Geo. E. Rogers, Agent, Rowlesburg, W. Va. Guarantee: (As above). Found: Phosphoric acid, soluble, 3.85 ; reverted, 3.48 ; insoluble, I.04: total, 8.37 ; available, 7.33 ; nitrogen, 0.94 ; potash, I. 34 ; availability of nitrogen, 75 ; chlorine, 5.9 .

Remark: Chlorine excessive.

6I5I. Bradley's Niagara Phosphate, Thompson \& Ireland, Agent, Worthington, W. Va. Guarantee: (As above). Found: Phosphoric acid, soluble, 3.24 ; reverted, 4.40 ; insoluble, I.o6; total, 8.70; available, 7.64; nitrogen, 0.9I ; potash, I.25; availability of nitrogen, 77 ; chlorine, 7.2.

Remark: Chlorine excessive.

6r48. Bradley's Soluble Dissolved Bone, Thompson \& Ireland, Agent, Worthington, W. Va. Guarantee: Phosphoric acid, soluble, I2 ; reverted, 2 ; insoluble, I ; total, I 5 ; available, I4; phosphoric acid from dissolved phosphate rock. Found: Phosphoric acid, soluble, I0.40; reverted. 4.47 ; insoluble, I.07; total, I 5.94; available, I4.87.

6r77. Bradley's Alkaline Bone \& Potash, Thompson \& Ireland, Agent, Worthington, W. Va. Guarantee: Phosphoric acid, soluble, 8 ; reverted, 2 ; insoluble, I ; total, II ; available, IO; potash, 2; phosphoric acid from dissolved phosphate rock, potash from kainit. Found: Phosphoric acid, soluble, 4.86; reverted, 5.09; insoluble, 0.69: total, Io.64; available, 9.95; potash, 2.07 ; chlorine. 5 .

Remark: Chlorine excessive.

6r86. Bradley's Dissolved Bone with Potash, Thos. Nuzum, Agent, Grafton, W. Va. Guarantee: Phosphoric acid, 
soluble, 6; reverted, 2 ; insoluble, I ; total, 9; available, 8; nitrogen, I.O3; potash, 2 ; phosphoric acid from dissolved animal bone and dissolved phosphate rock, nitrogen from animal tankage, potash from kainit. Found: Phosphoric acid, soluble, 5.86; reverted, 2.6r ; insoluble, I.02; total, 9.49 ; available, 8.47 ; nitrogen, I.05; potash, 2.32 ; availability of nitrogen, 73 ; chlorine, 4.I.

Remark: Chlorine excessive.

6036. Canton Chemical Potato \& Tobacco Manure, R. W. Sovine, Agent, Hurricane, W. Va. Guarantee: Phosphoric acid, soluble, 4 ; reverted, 2 ; insoluble, I ; total, 7 ; available, 6; nitrogen, I.24; potash, 5; phosphoric acid from dissolved animal bone and dissolved phosphate rock, nitrogen from animal tankage, potash, $1 / 4$ to $3 / 4$ from manure salt, $1 / 4$ to $3 / 4$ from kainit. Found: Phosphoric acid, soluble, 4.80 ; reverted, $3.9 \mathrm{I}$; insoluble, 2.09; total, Io.80 ; available, 8.7 I ; nitrogen, I.07 ; potash, 5.92 ; availability of nitrogen, 84 ; chlorine, I.I.

Remark: Nitrogen low.

6og8. Canton Chemical Potato Manure, Siever Hardware Co., Agent, Keyser, W. Va. Guarantee: (As above). Found: Phosphoric acid, soluble, 3.or ; reverted, 3.76; insoluble, I.43; tota1, 8.20; available, 6.77 ; nitrogen, I.44; potash, 5.39: availability of nitrogen, $8 \mathrm{I}$; chlorine, 2.6.

Remark: Source of potash equivalent to kainit.

6I29. Canton Chemical Potato \& Tobacco Manure, Washington, Alexander \& Cooke, Agents, Charlestown, W. Va. Guarantee: (As above). Found: Phosphoric acid, soluble, 3.77 ; reverted, 3.10 ; insoluble, 0.74 ; total, 7.61 ; available, 6.87 ; nitrogen. I.26; potash, 5.38 ; availability of nitrogen, 78 ; chlorine, 2.6 .

Remark: Source of potash equivalent to kainit.

6097. Canton Chemical Baker's Special Wheat, Corn and Grass Mixture, Siever Hardware Co., Agent, Keyser, W. 
Va. Guarantee: Phosplioric acid, soluble, 7; reverted, 2; insoluble, I; total, Io; available, 9; nitrogen, o.82; potash, 2 ; phosphoric acid from dissolved animal bone and disscived phosphate rock, nitrogen from animal tankage, potash from kainit. Found: Phosphoric acid, soluble, 5.30; reverted, 3.I incoluble, 1.24; total, IO.7I ; available, 9.47; nitrogen, I.II ; potash, 2.21 : availability of nitrogen, 74 ; chlorine, 2.9 .

6r25. Canton Chemical Baker's Special Wheat, Corn and Grass Mixture, Washington, Alexander \& Cooke, Agents, Charlestown, W. Va. Guarantee: (As above). Found: Phosphoric acid, soluble, 5.4I : reverted, 4.25: insoluble, I.20; total, Io.86; available, 9.66: nitrogen, 0.90; potash, I.96: availability of nitrogen, 69 ; chlorine, 2.9 .

6099. Canton Chemical Baker's Dissolved S. C. Bone, Siever Hardware Co., Agent, Keyser, W. Va. Guarantee: Phosphoric acid, soluble, I2; reverted, 2 ; insoluble, I ; total. I5: available, I4; phosphoric acid from dissolved phosphate rock. Found: Phosphoric acid, I0.94; reverted, 4.52 ; insoluble, I.I 5 : total, I6.6r : available, I 5.46.

6г26. Canton Chemical Baker's Dissolved S. C. Bone, Washington, Alexander \& Cooke, Agents, Charlestown, W. Va. Guarantee: (As above). Found: Phosphoric acid, soluble, 9.58 ; reverted, 4.26 ; insoluble, I.I8; total, r6.02 ; available, I 4.84 .

6r47. Canton Chemical Baker's Dissolved S. C. Bone, J. M. Haggerty, Agent, Farmington, W. Va. Guarantee: (As above). Found: Phosphoric acid, soluble, I0.63; reverted, 4.99; insoluble, I.Io; total, I6.72; available, I5.62.

6rог. Canton Chemical Harrow Brand Crop Grower, T. P. Licklider, Agent, Martinsburg, W. Va. Guarantee: Phosphoric acid, soluble, 6; reverted, 2 ; insoluble, I; tota1, 9; available, 8 ; nitrogen, 0.82 ; potash, I; phosphoric acid from 
dissolved animal bone and dissolved phosphate rock, nitrogen. from animal tankage, potash from kainit. Found: Phosphoric acid, soluble, 4.43 ; reverted, 3.82 ; insoluble, I.45; total, 9.70 ; available, 8.25 ; nitrogen, 0.99 ; potash, I.I2; availability of nitrogen, 78 ; chlorine, 7.x.

Remark: Chlorine excessive.

6145. Canton Chemical Game Guano, J. M. Haggerty, Agent, Farmington, W. Va. Guarantee: Phosphoric acid, soluble, 6 ; reverted, 2 ; insoluble, I; total, 9; available, 8; nitrogen, 1.65; potash, 2; phosphoric acid from dissolved animal bone and dissolved phosphate rock, nitrogen from animal tankage, potash from kainit. Found: Phosphoric acid, soluble, 5.26; reverted, 3.72 ; insoluble, I.69; total, I0.67; available, 8.98 ; nitrogen, I.8I : potash, 2.69 ; availability of nitrogen, 83 : chlorine, 2.7 .

6207. Canton Chemical Eagle Phosphate, T. P. Licklider, Agent, Martinsburg, W. Va. Guarantee: Phosphoric acid. soluble, 5 ; reverted, 2 ; insoluble, I; total ,8; available, 7 ; nitrogen, 0.82 ; potash, I; phosphoric acid from dissolved animal bone and dissolved phosphate rock, nitrogen from animal tankage, potash from kainit. Found: Phosphoric acid, soluble, 4.I2 ; reverted, 2.98; insoluble, I.46; tota1, 8.56; available, 7.I0; nitrogen, 0.99; potash, I.34; availability of nitrogen, 83 ; chlorine, 6.7 .

Remark: Chlorine excessive.

6048. Cleveland Dryer XXX Phosphate. West Charleston Feed Co., Agent, Charleston, W. Va. Guarantee: Phosphoric acid soluble, I2; reverted, 2; insoluble. I; total. I5: available, I4; phosphoric acid from dissolved phosphate rock. Found: Phosphoric acid, soluble, II.4I; reverted, 3.68 : insoluble, 2.62; total, I7.7I ; available, I 5.09.

6i62. Cleveland Dryer Forest City Buckeye, Bishop \& Barbe, Agent, Jane Lew, W. Va. Grarantee: Phosphoric acid, 
soluble, 7 ; reverted, 2 ; insoluble, I; total, Io; available, 9 ; nitrogen, 2.47; potash, 2 ; phosphoric acid from dissolved animal bone and dissolved phosphate rock, nitrogen from animal tankage, potash, $1 / 4$ to $3 / 4$ manure salts, $1 / 4$ to $3 / 4$ kainit. Found: Phosphoric acid, soluble, 5.27; reverted, 3.70 ; insoluble, I.39; total, I0.36; available, 8.97 ; nitrogen, 2.90 ; potash, 2.38; availability of nitrogen, 92 ; chlorine, I.9.

6r65. Cleveland Dryer Horsehead Phosphate \& Potash. Bishop \& Barbe, Agent, Jane Lew, W. Va. Guarantee: Phosphoric acid, soluble, 8 ; reverted, 2 ; insoluble. I : total. II : available, IO; potash, 2 ; phosphoric acid from dissolved phosphate rock, potash from kainit. Found: Phosphoric acid, soluble, 6.29; reverted, $3.6 \mathrm{r}$; insoluble, 0.65 ; total. I0.55: available, 9.90 ; potash, 2.27 ; chlorine, 6.3 .

Remark: Chlorine excessive.

6229. Cleveland Dryer Horsehead Phosphate. Chester Hardware Co., Agent, Chester, W. Va. Guarantee: Phosphoric acid, soluble, 8 ; reverted, 2 ; insoluble. I ; total, II ; available, Io; phosphoric acid from dissolved phosphate rock. Found: Phosphoric acid, soluble, 6.42; reverted, 5.23: insoluble, 2. I 5 ; total, I 3.80 : available, i .65.

6033. Detrick's Kangaroo Komplete Kompound, W. H. McCallister, Agent, Hurricane, W. Va. Guarantee: Phosphoric acid, soluble, 6; reverted, 2; insoluble, I : total, 9; available, 8 ; nitrogen, I.65; potash, 3 ; phosphoric acid from dissolved animal bone and dissolved phosphate rock, nitrogen from animal tankage, potash, $1 / 4$ to $3 / 4$ from manure salts, $\mathrm{I} / 4$ to $3 / 4$ kainit. Found: Phosphoric acid, soluble, 5.3I ; reverted, 4.90; insoluble, I.85; total, I2.06; available, I0.2I ; nitrogen, 1.65; potash, 2.42, availability of nitrogen, 85 : chlorine, 0.9 .

Remark: Potash low. 
6240. Detrick's Kangaroo Komplete Kompound, J. M. Miller \& Bro., Agent, Ronceverte, W. Va. Guarantee: (As above). Found: Phosphoric acid, soluble, 6.19; reverted, 3.17; insoluble, I.I3; total, I0.49; available, 9.36; nitrogen, 1.76; potash, 3.21 ; availability of nitrogen, 75 ; chlorine, 2.5 .

6034. Detrick's Imperial Compound, W. H. McCallister, Agent, Hurricane, W. Va. Guarantee: Phosphoric acid, soluble, 6 ; reverted, 2 ; insoluble, 1 ; total, 9 ; available, 8 ; nitrogen, 0.82 ; potash, 2 ; phosphoric acid from dissolved animal bone and dissolved phosphate rock, nitrogen from animal tankage, potash from kainit. Found: Phosphoric acid, soluble, 4; reverted, 4.23; insoluble, 2.29; total, ro.52; available, 8.23 ; nitrogen, 0.97 ; potash, 1.91 ; availability of nitrogen, 77 ; chlorine, I.I.

6r69. Detrick's Corn \& Oats Fertilizer, IV. S. Corrothers. Agent, Little Falls, W. Va. Guarantee: Phosphoric acid, soluble, 7 ; reverted, 2 ; insoluble, I ; total, ro; available, 9 ; nitrogen, 0.82 ; potash, 3 ; phosphoric acid from dissolved animal bone and dissolved phosphate rock, nitrogen from animal tankage, potash, $\mathrm{T} / 4$ to $3 / 4$ manure salts, $1 / 4$ to $3 / 4$ kainit. Found: Phosphoric acid, soluble, 6.70; reverted, 2.71 ; insoluble, 1.28: total, 10.69: available, 9.41 : nitrogen, 0.92 ; potash, 3.54: availability of nitrogen, 72 ; chlorine, 2.2.

6170. Detrick's Paragon Ammoniated Bone Phosphate \& Potash, W. S. Corrothers, Agent, Little Falls, W. Va. Guarantee: Phosphoric acid, soluble, 5; reverted, 2; insoluble, I ; total, 8; available, 7 ; nitrogen, 0.82 ; potash, I; phosphoric acid from dissolved animal bone and dissolved phosphate rock, nitrogen from animal tankage, potash from kainit. Found: Phosphoric acid, soluble, 4.I2; reverted, 3.37 ; insoluble, 0.92 ; total, 8.4I ; available, 7.49: nitrogen, o.9r : potash, T.27; availability of nitrogen, 75 ; chlorine, 6.3 .

Remark: Chlorine excessive. 
6r7I. Detrick's Dissolved S. C. Bone. II. S. Corrothers. Agent, Little Falls, W. Va. Guarantee: Phosphoric acid, soluble, I2; reverted, 2 : insoluble, I : total, I5; available. It; phosphoric acid from dissolved phosphate rock. Found: Phosphoric acid, soluble, IO.2I ; reverted, 4.89; insoluble. I.15: total, I6.25; available, I 5.ro.

6060. Great Eastern High Grade Bone \& Potash, E. R. Judy, Agent, Lewisburg, W. Va. Guarantee: Phosphoric acid, soluble, 8; reverted, 2 ; insoluble, I ; total, II ; available. Io; potash, 5 : phosphoric acid from dissolved phosphate rock, potash, $I / 4$ to $3 / 4$ manure salts; $I / 4$ to $3 / 4$ muriate of potash. Found: Phosphoric acid, soluble, 4.89; insoluble. 0.84; reverted, 5.64; total, II.37; available, I0.53; potash, 5.18; chlorine, I.9.

Remark: Source of potash equivalent to manure salts.

6ri8. Great Eastern High Grade Bone \& Potash, I. D. Anderson, Agent, Ronceverte, W. Va. Guarantee: (As above). Found: Phosphoric acid, soluble, 4.77 ; reverted, 4.74 ; insoluble, I.05; total, I0.56; available, 9.51 ; potash, 5 . 4 : chlorine, 2.I.

Remark: Phosphoric acid low.

6238. Great Eastern High Grade Bone \& Potash. Coleman \& Gribbs, Agent, Farmington, W. Va. Guarantee: (As above). Found: Phosphoric acid, soluble, 5.53: reverted. 4.63 ; insoluble, I.or ; total, I I.I7; available, Io.I6; potash, 5.85 ; chlorine, 2.3 .

Remark: Chlorine excessive.

6062. Great Eastern English Wheat Grower, E. R. Judy, Agent, Lewisburg, W. Va. Guarantee: Phosphoric acid, soluble, 6 ; reverted, 2 ; insoluble, I ; total, 9 ; available, 8 ; nitrogen, 0.82 ; potash, 2; phosphoric acid from dissolved animal bone and dissolved phosphate rock, nitrogen from animal 
tankage, potash from kainit. Found: Phosphoric acid, soluble, 4.49 ; reverted, 3.98 ; insoluble, 0.87 ; total, 9.34 ; available, 8.47 ; nitrogen, I.02; potash, 2.28; availability of nitrogen, $8 \mathrm{I}$. chlorine, 3.I.

Remark: Chlorine excessive.

6r 20. Great Eastern Corn Fertilizer, J. D. Anderson. Agent, Ronceverte, W. Va. Guarantee: Phosphoric acid, soluble, 6 ; reverted, 2 ; insoluble, I ; total, 9 ; available, 8 ; nitrogen, 0.82 ; potash, 4; phosphoric acid from dissolved animal bone and dissolved phosphate rock; potash, $1 / 4$ to $3 / 4$ manure salts, $1 / 4$ to $3 / 4$ kainit; nitrogen from animal tankage. Found: Phosphoric acid, soluble, 5.09; reverted, 3.72; insoluble, 0.9I ; total, 9.72 ; available, 8.8I ; nitrogen, 0.95 ; potash, 4.21 ; availahility of nitrogen, 72 ; chlorine, 2.

6I38. Great Eastern Corn Fertilizer, C. E. Casler, Agent, Berkeley Springs, W. Va. Guarantee: (As above). Found: Phosphoric acid, soluble, 4.55; reverted, 5.09; in-。 soluble, I.34; total, ro.98; available, 9.64 ; nitrogen, 0.87 : potash, 4.68; availability of nitrogen, 73 ; chlorine, 3.4 .

Remark: Source of potash equivalent to kainit. Chlorine excessive.

6I46. Great Eastern Corn Fertilizer, Coleman \& Gribbs, Agent, Farmington, W. Va. Guarantee: (As above). Found: Phosphoric acid, soluble, 3.53; reverted, 5.I5; insoluble, r.36: total, I0.04; available, 8.68 ; nitrogen, 1.02 : potash, 5.44 ; availability of nitrogen, 74 : chlorine, 2.2 .

6I2I. Great Eastern Dissolved Bone, J. D. Anderson. Agent, Ronceverte, W. Va. Guarantee: Phosphoric acid. soluble, I2 ; reverted, 2 ; insoluble, I; total, I5; available, I4: phosphoric acid from dissolved phosphate rock. Found: Phosphoric acid, soluble, Ir.86; reverted, 4.78 ; insoluble. 0.41 : total, I7.05; available, I6.64. 
6.14I. Great Eastern Dissolved Bone, C. E. Casler, Agent, Berkeley Springs, W. Va. Guarantee: (As above). Found: Phosphoric acid, soluble, 9.98; reverted, 5.5I; insoluble, I.24; total, I6.73; available, I5.49.

6135. Great Eastern Soluble Bone \& Potash, C. E. Casler, Agent, Berkeley Springs, W. Va. Guarantee: Phosphoric acid, soluble, 8; reverted, 2 ; insoluble, I ; total, II ; available. Io; potash, 2 ; phosphoric acid from dissolved phosphate rock, potash from kainit. Found: Phosphoric acid, soluble, 6.06: reverted, 5.Io; insoluble, 0.69; total, II.85; available, II.I6: potash, 2.01 ; chlorine, 5 .

Remark: Chlorine excessive.

6r50. Great Eastern Japanese Wheat Grower, Coleman \& Gribbs, Agent, Farmington, IV. Ta. Guarantee: Phosphoric acid, soluble, 4; reverted, 2 ; insoluble, 1 ; total, 7 ; available, 6; potash, 3 ; phosphoric acid from dissolved phosphate rock; potash from kainit. Found: Phosphoric acid, soluble, 2.15 ; reverted, 4.56 ; insoluble, 0.54 ; total, 7.25 ; available, 6.7I ; potash, 5.20; chlorine, 3.I.

Remark: Chlorine excessive.

6228. Great Eastern Japanese Wheat Grower, Coleman \& Gribbs, Farmington, W. Va. Guarantee: (As above). Found: Phosphoric acid, soluble, 3.6r ; reverted, 3.26; insoluble, o.I3; total, 7 ; available, 6.87; potash, 3.58 ; chlorine, 5.2 .

Remark: Chlorine excessive.

6083. Lazaretto Special Potato \& Tobacco Fertilizer. T. B. Drummond \& Co., Agent, Buckhannon, W. Va. Guarantee: Phosphoric acid, soluble, 6; reverted, 2 ; insoluble. I: total, 9; available, 8 ; nitrogen, o.82; potash, 4; phosphoric acid from dissolved animal bone and dissolved phosphate rock; nitrogen from animal tankage; potash. $1 / 4$ to $3 / 4$ ma- 
nure salts, $1 / 4$ to $3 / 4$ kainit. Found: Phosphoric acid, solublé, 3.90 ; reverted, 4.70; insoluble, I.I5; total, 9.75 ; available, 8.60; nitrogen, 0.86 ; potash, 4.30 ; availability of nitrogen, 70 ; chlorine, 2.6 .

Remark: Source of potash equivalent to kainit.

6082. Lazaretto Dissolved Bone \& Phosphate, T. B. Drummond \& Co., Agent, Buckhannon, W. Va. Guarantee: Phosphoric acid, soluble, I2; reverted, 2; insoluble, I; total. I5; available, I4; phosphoric acid from dissolved phosphate rock. Found: Phosphoric acid, soluble, IO.50; reverted, 5.II ; insoluble, I.II; total, I6.72: available, I5.6г.

6I79. Maryland Tornado Fertilizer, Seslir \& Hawker Hdw. Co., Agent, Shinnston, W. Va. Guarantee: Phosphoric acid, soluble, Io; reverted, 2 ; insoluble, I ; total, I3; available, I2; potash, 5; phosphoric acid from dissolved phosphate rock; potash from muriate of potash. Found: Phosphoric acid, soluble, 9.54 ; reverted, $3.5 \mathrm{I}$; insoluble, 0.75 ; total, I3.80; available, I3.15; potash, 5.48 ; chlorine, 2.

Remark: Source of potash equivalent to manure salts.

6I80. Maryland's Dissolved S. C. Phosphate, W. S. Stout, Agent, Harrisville, W. Va. Guarantee: Phosphoricacid, soluble, I2 ; reverted, 2 ; insoluble, I; total, I5; available, I4; phosphoric acid from dissolved phosphate rock. Found: Phosphoric acid, soluble, I0.77; reverted, 4.74; insoluble, 0.99; total, I6.50; available, I 5.5I.

6225. Maryland's Dissolved S. C. Phosphate, W. S. Stout, Agent, Harrisville, W. Va. Guarantee: (As above). Found: Phosphoric acid, soluble, II.85; reverted, 3.96 ; insoluble, 0.23 ; total, I6.04; available, I5.8I.

6037. Williams \& Clark's Good Grower Potato Phosphate, A. G. Crislip, Agent, Philippi, W. Va. Guarantee: 
Phosphoric acid, soluble, 4; reverted, 2 ; insoluble, I; total, 7 ; available, 6; nitrogen, I.24; potash, 5; phosphoric acid from dissolved animal bone and dissolved phosphate rock; nitrogen from animal tankage. Potash, $1 / 4$ to $3 / 4$ from manure salts, $1 / 4$ to $3 / 4$ from kainit. Found: Phosphoric acid, soluble, 3.39 ; reverted, 3.46 ; insoluble, 1.47 ; total, 8.32 ; available, 6.85; nitrogen, I.44; potash, 5.I8; availability of nitrogen, 86; chlorine, 2.7 .

Remark: Source of potash equivalent to kainit.

6038. William's \& Clark's Acorn Acid Phosphate, A. G. Crislip, Agent, Philippi, W. Va. Guarantee: Phosphoric acid, soluble, I2; reverted, 2 ; insoluble, I ; total, I5; available, I4; phosphoric acid from dissolved phosphate rock. Found: Phosphoric acid, soluble, Io.62; reverted, 4.85 ; insoluble, 0.75 ; total, I6.22; available, I 5.47 .

6039. Williams and Clark's Prolific Fertilizer, A. G. Crislip, Agent, Philippi, W. Va. Guarantee: Phosphoric acid. soluble, 5 ; reverted, 2 ; insoluble, $I$; total, 8 ; available. 7 : nitrogen, 0.82 ; potash, I; phosphoric acid from dissolved animal bone and dissolved phosphate rock; nitrogen from animal tankage; potash from kainit. Found: Phosphoric acid. soluble, 3.72 ; reverted, 3.65 ; insoluble, 0.92 ; total, 8.29 ; available, 7.37 ; nitrogen, 0.92 : potash, I.I6; availability of nitrogen, $8 \mathrm{I}$; chlorine, 7 .

Remark: Chlorine excessive.

6226. Williams \& Clark's Royal Bone Phosphate, Wheeling Implement Buggy Co., Agent, Wheeling, W. Va. Guarantee: Phosphoric acid, soluble, 6; reverted, 2 ; insoluble, I t total, 9; available, 8 ; nitrogen, I.03; potash, 2 ; phosphoric, acid from dissolved animal bone and dissolved phosphate rock; nitrogen from animal tankage; potash from kainit. Found: Phosphoric acid, soluble, 4.68; reverted, 3.97 ; insoluble, I.98; total, Io.63: available, 8.65 ; nitrogen, I.I2 ; po乞ash, 2.44; availability of nitrogen. 79 , chlorine, 2.9 . 
6236. Southern Fertilizer Co.'s Bone \& Potash Mixture, W. C. Hovemale \& C. A. Rider, Agents, Berkeley Springs, W. Va. Guarantee: Phosphoric acid, soluble, 6; reverted, 2; insoluble, I ; total, 9; available, 8; potash, 2; phosphoric acid from dissolved phosphate rock; potash from kainit. Found: Phosphoric acid, soluble, 4.63 ; reverted, 4.02; insoluble, 0.32 ; total, 8.97 ; available, 8.65 ; potash, 2.56 ; chlorine, 7.2 .

Remark: Chlorine excessive.

6224. Susquehanna Superior Rock Phosphate, W. N. Lemen \& Son, Agent, Shephardstown, W. Va. Guarantee: Phosphoric acid, soluble, I2; reverted, 2; insoluble, I; total, I5; available, I4; phosphoric acid from dissolved phosphate rock. Found: Phosphoric acid, soluble, 9.44; reverted, 5.52; insoluble, 0.83 ; total, I5.79; available, I4.96.

6023. Zell's Dissolved Bone Phosphate, Cicero Phillips, Agent, Belington, W. Va. Guarantee: Phosphoric acid, soluble, I2 ; reverted, 2 ; insoluble, I; total, 15 ; available, I4. Found: Phosphoric acid, soluble, I0.95; reverted, 4.57 ; insoluble, I.OI ; total, I6.53; available, I5.52.

6i i4. Zell's Dissolved Bone Phosphate, Exchange Mill Co., Agent, Grafton, W. Va. Guarantee: (As above). Found: Phosphoric acid, soluble, 9.45 ; reverted, 5.53; insoluble, r.or ; total, I5.99; available, I4.98.

6024. Zell's Electric Phosphate, Cicero Philipps, Agent, Belington, W. Va. Guarantee: Phosphoric acid, soluble, 8; reverted, 2; insoluble, I ; total, II ; available, IO; potash, 2; phosphoric acid from dissolved phosphate rock; potash from kainit. Found: Phosphoric acid, soluble, 6.17; reverted, 4.4I ; insoluble, 0.54 ; total, II.I2; available, I0.58; potash, 2.20; chlorine, 5.I.

Remark: Chlorine excessive. 
6063. Zell's Electric Phosphate, Ira J. Curry, Agent, Grafton, W. Va. Guarantee: (As above). Found: Phosphoric acid, soluble, 6.28; reverted, 4.23 ; insoluble, 0.67 ; total, II.I8; available, I0.4r ; potash, 2.I8; chlorine, 5.I.

Remark: Chlorine excessive.

6035. Zell's Little Giant, Mossman Bros., Agent, Huntington, W. Va. Guarantee: Phosphoric acid, soluble, 5; reverted, 2 ; insoluble, I; total, 8; available, 7 ; nitrogen, o.S2: potash, I; phosphoric acid from dissolved animal bone and dissolved phosphate rock; nitrogen from animal tankage: potash from kainit. Found: Phosphoric acid, soluble, r.66: reverted, 5.20; insoluble, 2.65; total, 9.5I; available, 6.86; nitrogen, I.05; potash, I.I7; availability of nitrogen, 77 ; chlorine, I.2.

Remark: Phosphoric acid low.

6r34. Zell's Little Giant, R. Hunter, Agent, Berkeley Springs, W. Va. Guarantee: (As above). Found: Phosphoric acid, soluble, 3.9I; reverted, 4.67; insoluble, I.09; total. 9.67; available, 8.58 ; nitrogen, 0.99 ; potash, I.19; availability of nitrogen, 78 ; chlorine, 6.8 .

Remark: Chlorine excessive.

6064. Zell's Special Compound for Potatoes and Vegetables, Ira J.' Curry ,Agent, Grafton, W. Va. Guarantee: Phosphoric acid, soluble, 6; reverted, 2; insoluble, I; total, 9; available, 8 ; nitrogen, 2.47 ; potash, 4 ; phosphoric acid from dissolved animal bone and dissolved phosphate rock; nitrogen from animal tankage; potash from muriate of potash. Found: Phosphoric acid, soluble, 5.37; reverted, 3.84; insoluble, I.79; total, I I ; available, 9.21 ; nitrogen, 2.52; potash, 4.39: availability of nitrogen, 93 ; chlorine, r.5.

Remark: Source of potash equivalent to manure salts.

6ri3. Zell's Economizer Phosphate, Exchange Mill Co.. Agent. Grafton, W. Va. Guarantee: Phosphoric acid, solu- 
ble, 6; reverted, 2 ; insoluble, I; total, 9; available, 8 ; nitrogen, 0.82 ; potash, 2 ; phosphoric acid from dissolved animal bone and dissolved phosphate rock; nitrogen from animal tankage; potash from kainit. Found: Phosphoric acid, soluble, 5.I2 ; reverted, 3.52 insoluble, I.24; total, 9.88; available. 8.64 ; nitrogen, I.09; potash, 2.42 ; availability of nitrogen, 77 ; chlorine, 3.7 .

Remark: Chlorine excessive.

6227. Zell's Ammoniated Bone Super-Phosphate, R. Hunter, Agent, Berkeley Springs, W. Va. Guarantee: Phosphoric acid, soluble, 6; reverted, 2 ; insoluble, I; total, 9: available, 8 ; nitrogen, I.65; potash, 2 : phosphoric acid from dissolved animal bone and dissolved phosphate rock; nitrogen from animal tankage; potash from kainit. Found: Phosphoric acid, soluble, 5.30; reverted, 3.69; insoluble, I.64; total, I0.63; available, 8.99 ; nitrogen, I.88; potash, 2.61 ; availability of nitrogen, 84 ; chlorine, 2.9.

\section{THE ARMOUR FERTILIZER WORKS,}

Baltimore, Md., and Buena Vista, Virginia.

6032. Grain Grower, Myne \& Green, Agent, Hurricane, W. Va. Guarantee: Phosphoric acid, soluble, 6; reverted, 2: insoluble, 2 ; total, Io; available, 8 ; nitrogen, 1.65 ; potash, 2 : phosphoric acid from animal bone and acid phosphate; nitrogen, I-Io to 2-Io bone, 8-1o to 9-Io bone tankage; potash, $1 / 5$ to $2 / 5$ muriate, $3 / 5$ to $4 / 5$ kainit. Found: Phosphoric acid, soluble, 4.4I ; reverted, 3.89; insoluble, 0.87 ; total, 9.17; available, 8.30 ; nitrogen, 1.69; potash, 2.29; availability of nitrogen. 93; chlorine, 3.6.

Remark: Source of potash equivalent to kainit. Chlorine excessive.

6105. Grain Grower, R. N. Stewart \& Son, Agent, Martinsburg, W. Va. Guarantee: (As above). Found: Phos- 
phoric acid, soluble, 4.99; reverted, 3.01 ; insoluble, 0.5 r total, $8.5 \mathrm{I}$; available, 8 ; nitrogen, I.48; potash, 2.23 ; availability of nitrogen, 83 ; chlorine, 3.2 .

Remark: Source of potash equivalent to kainit. Nitrogen low. Chlorine excessive.

6061. Royal Ammoniated Bone, E. R. Judy, Agent, Lewisburg, W. Va. Guarantee: Phosphoric acid, soluble, 6; reverted, 2 ; insoluble, 2 ; total, Io; available, 8 ; nitrogen, 0.82 ; potash, 4; phosphoric acid from acid phosphate and animal bone, nitrogen, $1 / 5$ to $3 / 5$ from bone; $2 / 5$ to $4 / 5$ bone tankage; potash, $1 / 3$ to $2 / 3$ from kainit; $1 / 3$ to $2 / 3$ from muriate. Found: Phosphoric acid, soluble, 5.21 ; reverted, 3.73 ; insoluble, 1.42; total, ro.36; available, 8.94; nitrogen, 0.69; potash, 4.96; availability of nitrogen, $7 \mathrm{I}$; chlorine, 2.3 .

Remark: Nitrogen low.

6210. Royal Ammoniated Bone. R. N. Stewart \& Son, Aegnt, Martinsburg, W. Va. Guarantee: (As above). Found: Phosphoric acid, soluble, 5.63; reverted, 2.36; insoli1ble, 0.68; total, 8.67; available, 7.99; nitrogen, 0.84 ; potash, 4.08: availability of nitrogen, 74 ; chlorine, 1.9.

6ro6. Phosphate and Potash, No. I, R. N. Stewart \& Son, Agent, Martinsburg, W. Va. Guarantee: Phosphoric acid, soluble, 8 ; reverted, 2 ; insoluble, 2 ; total, I2 ; available, Io; potash, 2; phosphoric acid from acid phosphate, potash, $1 / 5$ to $2 / 5$ from muriate; $3 / 5$ to $4 / 5$ kainit. Found: Phosphoric acid, soluble, 6.86; reverted, 2.37 ; insoluble, 0.84; total, 10.07; available, 9.23; potash, 2.16; chlorine, 3.2.

Remark: Phosphoric acid low. Source of potash equivalent to kainit.

6ro7. Phosphate and Potash, No. 2, R. N. Stewart \& Son, Agent, Martinsburg, W. Va. Guarantee: Phosphoric acid, soluble, 6; reverted, 2; insoluble, I; total, 9; available, 8; 
potash, 5; phosphoric acid from acid phosphate; potash, 2/5 to $3 / 5$ muriate of potash; $2 / 5$ to $3 / 5$ kainit. Found: Phosphoric acid, soluble, 5.I6; reverted, 2.70 ; insoluble, 0.60 ; total, 8.46; available, 7.86; potash, 4.79; chlorine, 3.2.

Remark: Source of potash equivalent to kainit. Chlorine excessive.

6io8. Star Phosphate, R. N. Stewart \& Son, Agent, Martinsburg, W. Va. Guarantee: Phosphoric acid, soluble, I2 ; reverted, 2 ; insoluble, 2 ; total, I6; available, I4; phosphoric acid from acid phosphate. Found: Phosphoric acid. soluble, 12.84 ; reverted, 2.22 ; insoluble, 0.15; total, I5.21; available, 15.06 .

6or3. Star Phosphate, submitted for analysis by I. D. Bowman, Hoult, W. Va. Guarantee: (As above). Found: Phosphoric acid, soluble, I3.34; reverted, I.98; insoluble, 0.47 ; total, 15.79 ; available, I 5.32 .

6I53. Star Phosphate, W. H. Bailey Hardware Co.. Agent, Morgantown, W. Va. Guarantee: (As above). Found: Phosphoric acid, soluble, I3.30; reverted, 2.33: insoluble, 0.26 ; total, I5.89; available, I 5.63.

6I57. Phosphate and Potash, No. 2, W. H. Bailey Hardware Co., Agent, Morgantown, W. Va. Guarantee: Phosphoric acid, soluble, 6; reverted, 2 ; insoluble, I ; total, 9; available, 8; potash, 5 ; phosphoric acid from acid phosphate, potash, $2 / 5$ to $3 / 5$ from kainit; $2 / 5$ to $3 / 5$ muriate. Found: Phosphoric acid, soluble, 5.04 ; reverted, 3.75 ; insoluble, 0.70 : tota1, 9.49; available, 8.79; potash, 5.03; chlorine, 3 .

Remark: Source of potash equivalent to kainit.

6202. Wheat Special, Geo. B. Hooff, Agent, Charles Town, W. Va. Guarantee: Phosphoric acid, soluble. 6; reverted, 2 ; insoluble, 2 : total, Io: available, 8 ; nitrogen, 0.82 ; 
potash, 2; phosphoric acid from acid phosphate and animal bone, nitrogen, $1 / 5$ to $2 / 5$ bone; $3 / 5$ to $4 / 5$ bone tankage; potash from kainit. Found: Phosphoric acid, soluble, 6.04; reverted, 2.78; insoluble, 0.65 ; total, 9.47 ; available, 8.82 ; nitrogen, 0.72 ; potash, 2.03; availability of nitrogen, 72 ; chlorine, 3.3 .

Remark: Nitrogen low. Source of potash equivalent to kainit. Chlorine excessive.

6230. Bone Meal, Bishop \& Barbe, Agent, Jane Lew, W. Va. Guarantee: Phosphoric acid, total, 24; nitrogen, 2.47; phosphoric acid from animal bone, nitrogen from bone. Found: Phosphoric acid, total, 24.60; nitrogen, 2.43: availability of nitrogen, 89 .

6or4. Muriate of Potash, submitted for analysis by J. D. Bowman, Hoult, W. Va. Found: Potash, 48; chlorine, 0.9.

\section{BAUGH \& SONS CO.,}

Philadelphia, Pa., and Norfolk, Va.

6025. Bangh's Excelsior Guano, West Virginia Implement Co., Agent, Elkins, W. Va. Guarantee: Phosphoric acid, insoluble, 2 ; total, Io ; available, 8 : nitrogen, 0.823 ; potash, 4; phosphoric acid from phosphate rock; nitrogen, $1 / 2$ from animal tankage; $1 / 2$ from garbage tankage; potash, $1 / 2$ from muriate of potash; $1 / 2$ from kainit. Found: Phosphoric acid, soluble, 6.I7 ; reverted, 3.59; insoluble, 0.79; tota1, ro.55; available, 9.76 ; nitrogen, I.30; potash, 4.49: availability of nitrogen, 80; chlorine, r.9.

6094. Batgh's Excelsior Guano, Dean \& Reeves, Fairmont, W. Va. Guarantee: (As above). Found: Phosphoric acid, soluble, 3.42 ; reverted. 5.I3: insoluble, I.30; total, 9.85; available. 8.55 ; nitrogen, 1.03 ; potash, 4.97 : availability of nitrogen, 68; chlorine, 2.2.

Remark: Chlorine excessive. 
6026. Baugh's Special Potato Manure, West Virginia Implement Co., Agent, Elkins, W. Va. Guarantee: Phosphoric acid, insoluble, 2 ; total, 7 ; available, 5 ; nitrogen, 1.65; potash, IO; phosphoric acid from phosphate rock; nitrogen, $1 / 2$ from animal tankage; $1 / 2$ from sulphate of ammonia; potash from muriate of potash. Found: Phosphoric acid, soluble, 2.79; reverted, 3.09 ; insoluble, 0.52 ; total, 6.40; available, 5.88 ; nitrogen, 1.94; potash, 10.98; availability of nitrogen, 91; chlorine, I.5.

Remark: Source of potash equivalent to manure salts.

6o68. Baugh's Special Potato Manure, N. C. Musgrave, Agent, Grafton, W. Va. Guarantee: (As above). Found: Phosphoric acid, soluble, 4 ; reverted, $1.5 \mathrm{I}$; insoluble, 0.45 ; total, 5.96 ; available, 5.51 ; nitrogen, 1.83 ; potash, Io.80 availability of nitrogen, 9o; chlorine, r.6.

Remark: Source of potash equivalent to manure salts.

6095. Baughs' Special Potato Manure, Dean \& Reeves, Agent, Fairmont, W. Va. Guarantee: (As above). Found: Phosphoric acid, soluble 4,27; reverted, I.37; insoluble, 0.54; total, 6.18; available, 5.64; nitrogen, I.72; potash, ro.41 ; availability of nitrogen, 9I ; chlorine, I.5.

Remark: Source of potash equivalent to manure salts.

6027. Baugh's Export Bone with Potash, West Virginia Implement Co., Asent, Elkins, W. Va. Guarantee: Phosphoric acid, total, I I ; nitrogen, r.65; potash, 2 ; phosphoric acid from animal bone; nitrogen from animal bone: potasl. $T / 2$ from muriate, $1 / 2$ from kainit. Found: Phosphoric acid, total, Ir.78; nitrogen, I.89; potash, 3.18; availability of nitrogen, 95; chlorine, 3.9.

Remark:- Source of potash equivalent to kainit. Chlorine excessive.

6III. Baugh's Export Bone with Potash, T. B. Licklider, Agent, Martinsburg. W. Va. Guarantee: (As above). 
Found: Phosphoric acid, total, I2.72; nitrogen, 2.29: potash, 2.J3: availability of nitrogen, 95 ; chlorine, 3.5 .

Remark: Source of potash equivalent to kainit. Chlorine excessive.

6045. Baugh's High Grade Acid Phosphate, Philippi Mill Co., Agent, Philippi, IV. Va. Guarantee: Phosphoric acid, insoluble, I; total, I5; available, I4; phosphoric acid from dissolved rock. Found: Phosphoric acid, soluble, I3.I5; reverted, I.93: insoluble, O.I9; total, I5.27: available, I.5.08.

6073. Baugh's High Grade Acid Phosphate, Offutt \& Lakin, Terra Alta, W. Va. Guarantee: (As above). Found: Phosphoric acid, soluble, 9.49; reverted, 5.72 ; insoluble, o.6I; total, I 5.82 ; available, I5.21.

6047. Baugh's i2 \& 5, Philippi Mill Co., Agent, Philippi. WV. Va. Guarantee: Phosphoric acid, available, I2; potash, 5 ; phosphoric acid from dissolved rock; potash, $1 / 2$ from muriate of potash, $1 / 2$ from kainit. Found: Phosphoric acid, soluble, 6.32 ; reverted, 5.62 ; insoluble, 0.78 ; total, 12.72 ; available, II.94; potash, 4.89 ; chlorine, I.5.

6046. Baugh's Soluble Alkaline, Philippi Mill Co., Agent, Philippi, W. Va. Guarantee: Insoluble, I; total, II ; available, Io; potash, 2 ; phosphoric acid from dissolved rock; potash from kainit. Found: Phosphoric acid, soluble, I.I9; reverted, 8.93; insoluble, o.9r; total, Ir.o3; available, IO.I2; potash, 2.36; chlorine, 3.4 .

Remark: Chlorine excessive.

6074. Baugh's Soluble Alkaline, Offutt \& Lakin, Agent, Terra Alta, W. Va. Guarantee: (As above). Found: Phosphoric acid, soluble, 0.92 ; reverted, 9.08 ; insoluble, I.30; total, I I.30: available, Io; potash, 2.32 : chlorine, 2.9 . 


\section{8}

6065. Baugh's Animal Bone and Potash Compound, (for all crops), Conoway \& Clayton, Agents, Barracksville, W. $\checkmark$ a. Guarantee: Phosphoric acid, insoluble, 2 ; total, Io; available, 8 ; nitrogen, I.65; potash, 2 ; phosphoric acid from phosphate rock; nitrogen, $2 / 3$ from animal tankage, $1 / 3$ from sulphate of amonia; potash, $1 / 2$ from muriate of potash, $1 / 2$ from kainit. Found: Phosphoric acid, soluble, 5.63; reverted, 3.78; insoluble, I.I4; total, I0.55; available, $9.4 \mathrm{I}$; nitrogen, I.98; potash, 2.5I ; availability of nitrogen, 83 ; chlorine, 2.8 .

Remark: Source of potash equivalent to kainit.

6066. Baugh's Potato and Truck Special, Conoway \& Clayton, Agent, Barracksville, W. Va. Guarantee: Phosphoric acid, insoluble, 2; total, 9; available, 7 ; nitrogen, 2.88; potash, 7 ; phosphoric acid from phosphate rock; nitrogen, $1 / 2$ from animal tankage, $1 / 2$ from sulphate of ammonia; potash from muriate of potash. Found: Phosphoric acid, soluble, 6.37 ; reverted, 1.61 ; insoluble, o.32; total, 8.30; available. 7.98; nitrogen, 3.4I ; potash, 6.04; availability of nitrogen, 95 ; chlorine, I.8.

Remark: Potash low. Source of potash equivalent to manure salts.

6093. Baugh's Potato and Truck Special, Dean \& Reeves. Agent, Fairmont, W. Va. Guarantee: (As above). Found: Phosphoric acid, soluble, 5.OI: reverted, 3.I3; insoluble, 0.97 ; total, 9.II ; available, 8.I4; nitrogen, 2.90 ; potash, 7.82 : availability of nitrogen, 94; chlorine, I.2.

Remark: Chlorine excessive.

6067. Baugh's Double Eagle \$25 Phosphate Raw Bone Super Phosphate, N. C. Musgrave, Agent, Grafton, W. Va. Guarantee: Phosphoric acid, insoluble, 2; total, ro; available, 8 ; nitrogen, I.65; potash, I: phosphoric acid from Phosphate rock; nitrogen, $2 / 3$ from animal tankage, $1 / 3$ from sulphate of ammonia; potash from kainit. Found: Phosphoric acid, so- 
luble, 4.68 ; reverted, $4.8 \mathrm{I}$; insoluble, 1.23 ; total, 10.72 : available, 9.49 ; nitrogen, I.77; potash, I.50; availability of nitrogen. $8_{5}$; chlorine, 3 .

6ıo9. Baugh's Complete Aninal Bone Fertilizer, 'T. P. Licklider, Martinsburg, W. Va. Guarantee: Phosphoric acid, available, 8 ; nitrogen, I.65; potash, 5 ; phosphoric acid from phosphate rock; nitrogen, $2 / 3$ from animal tankage, $1 / 3$ sulphate of ammonia; potash, $1 / 2$ from muriate of potash, $1 / 2$ from kainit. Found: Phosphoric acid, soluble, 4.I8; reverted, 4.20; insoluble, o.6r ; total, 8.99 ; available, 8.38 ; nitrogen. I.88 ; potash, 4.80 ; availability of nitrogen, 92 ; chlorine, 1.6.

6i Io. Baugh's General Crop Grower, T. P. Licklider: Agent, Martinsburg; W. Va. Guarantee: Phosphoric acid, insoluble, 2 ; total, Io; available, 8 ; nitrogen, 0.823 ; potash, I; phosphoric acid from phosphate rock; nitrogen. $1 / 2$ from animal tankage, $1 / 2$ from garbage tankage; potash from kainit. Found: Phosphoric acid, soluble, 4.22; reverted, 4.42; insoluble, I.48; tota1, Io.I2; available, 8.64 ; nitrogen. I.I6; potash, I.35; availability of nitrogen, 77 ; chlorine, 2.8 .

6i 12. Baugh's Pure Dissolved Animal Bones, T. P. Iicklider, Agent, Martinsburg, W. Va. Guarantee: Phosphoric acid, insoluble, 3; total, I6: available, I3; nitrogen, 2.06; phosphoric acid from animal bone; nitrogen from animal bone. Found: Phosphoric acid, soluble, 7.02; reverted, 9.27; insoluble, o.8I ; total, I7.Io; available, I6.29; nitrogen, 2.20; availability of nitrogen, 90 .

6200. Special Mixture, G. T. Hodges, Agent, Shepherdstown, W. Va. Guarantee: Phosphoric acid, insoluble, 2 ; total, IO; available, 8; nitrogen, I.02; potash, I.25; phosphoric acid from phosphate rock: nitrogen, $2 / 3$ from animal tankage, $1 / 3$ sulphate of ammonia: potash. $1 / 2$ from muriate of potash. $1 / 2$ from kainit. Found: Phosphoric acid, soluble 4.17 : reverted 
7.20; insoluble, 2.48 ; total, 13.85 ; available, II.37; nitrogen, I.36; potash, 4.24; availability of nitrogen, 79; chlorine, 2.9.

Remark: Chlorine excessive.

620r. Long's Special Mixture, L. M. Long, Agent, Rippon, W. Va. Guarantee: Phosphoric acid, available, I3; nitrogen, I.23; potash, 2.50; phosphoric acid from phosphate rock; nitrogen, $1 / 3$ from dissolved animal bone, $1 / 3$ blood, $1 / 3$ animal tankage; potash from muriate of potash. Found: Phosphoric acid, soluble, I0.39; reverted, 3.6r ; insoluble, I.20; total, I 5.20 ; available, I4; nitrogen, I.35; potash, 2.84; availability of nitrogen, 9I ; chlorine, 0.8 .

6218. Baugh's Ammoniated Soluble Alkaline, G. T. Hodges, Agent, Shepherdstown, W. Va. Guarantee: Phosphoric acid, insoluble, 2 ; total, Io; available, 8 ; nitrogen, 0.4I ; potash, 2; phosphoric acid from phosphate rock; nitrogen from garbage tankage; potash, $1 / 2$ from kainit, $1 / 2$ muriate of potash. Found: Phosphoric acid, soluble, 2.87 ; reverted, 5.55 ; insoluble, I.ro; total, 9.52 ; available, 8.42 ; nitrogen, 0.49 ; potash, 3.06; availability of nitrogen, 50 ; chlorine, 2.9.

Remark: Source of potash equivalent to kainit.

6219. Baugh's Wheat Fertilizer, Conoway \& Clayton. Agent, Barracksville, W. Va. Guarantee: Phosphoric acid, insoluble, 2; total, Io; available, 8 ; nitrogen, I.65; potash, 2; phosphoric acid from phosphate rock: nitrogen, $2 / 3$ from animal tankage, $1 / 3$ sulphate of ammonia; potash, $1 / 2$ muriate of potash, $1 / 2$ kainit. Found: Phosphoric acid, soluble, 3.22; reverted, 6.06, insoluble, т.65; total, т0.93; available, 9.28; nitrogen, 2.16; potash, 2.62; availability of nitrogen, 89; chlorine, 2.6.

Remark: Source of potash equivalent to kainit. 
BOWKER FERTILIZER COMPANI.

Boston, New York and Cincinnati.

6085. Bowker's Bone Meal, T. B. Drummond \& Co., Agent, Buckhannon, W. Ta. Guarantee: Phosplhoric acid. total, 25; nitrogen, I.23; phosphoric acid from bone; nitrogen from bone. Found: Phosphoric acid, total, 27.20; nitrogen, 1.36: availability of nitrogen, 92.

6ogo. Bowker's Bone Meal, Orie Myers, Agent, Clarksburg, W. Va. Guarantee: (As above). Found: Phosphoric acid, total, 21.79; nitrogen, I.53; availability of nitrogen, 88 .

Remark: Phosphoric acid low.

6086. Bowker's Dissolved Bone and Potash, T. B. Drummond \& Co., Agent, Buckhannon, W. Va. Guarantee: Phosphoric acid, insoluble, 2; total, I2; available, Io; potash, 2; phosphoric acid from phosphate rock; potash from muriate. Found: Phosphoric acid, soluble, 6.08; reverted, 5.03 ; insoluble, 3.65 ; total, I4.76; available, II.II; potash, 2.73: chlorine, I.

6088. Bowker's ro percent Manure, Orie Myers, Agent, Clarksburg, W. Va. Guarantee: Phosphoric acid, insoluble. 2; total, 7 ; available, 5 ; nitrogen, 0.82 ; potash, Io: phosphoric acid from phosphate rock: potash from muriate; nitrogen from animal tankage. Found: Phosphoric acid, soluble, 2.96; reverted, 3.43 ; insoluble, 2.I4; total, 8.53; available, 6.39: nitrogen, $0.8 \mathrm{I}$; potash, II.45; chlorine, 9; availability of nitrogen, 76 .

6089. Bowker's Harvest Bone, Orie Myers, Agent, Clarksburg, W. Va. Guarantee: Phosphoric acid, insoluble, 2; total, Io; available, 8 ; nitrogen, 0.82 ; potash, I; phosphoric acid from phosphate rock; nitrogen from animal tankage; 
potash from muriate. Found: Phosphoric acid, soluble, 4.25; reverted, 4.96; insoluble, 2.47 ; total, Ir.68; available, 9.21 ; nitrogen, 0.96 ; potash, I.2I ; availability of nitrogen, 75 ; chlorine, .7 .

6r24. Bowker's Harvest Bone, J. M. Miller \& Bro,. Agent, Ronceverte, W. Va. Guarantee: (As above). Found: Phosphoric acid, soluble, 4.44: reverted, 4.64; insoluble, 2.24; total, II.32; available, 9.08; nitrogen, I.06; potash, I.og; availability of nitrogen, 82 ; chlorine, .9.

6159. Bowker's Harvest Bone, W. F. Sill, Agent, Pennsboro, W. Va. Guarantee: (As above). Found: Phosphoric acid, soluble, $4.7 \mathrm{I}$; reverted, 5.65 ; insoluble, 2.87 ; total, r3.23; available, I0.36; nitrogen, 0.94 ; potash, I.07; availability of nitrogen, 78 ; chlorine, 8 .

6i60. Bowker's Soluble Bone, W. F. Sill, Agent, Pennsboro, W. Va. Guarantee: Phosphoric acid, insoluble, 2; total, I6; available, I4; phosphoric acid from phosphate rock. Found: Phosphoric acid, soluble, I3.05; reverted, 3.67 ; insoluble, 2.06; total, I8.78; available, I6.72.

623I. Bowker's Soluble Bone, W. F. Sill, Agent, Pennsboro, W. Va. Guarantee: (As above). Found: Phosphoric acid, soluble, I 3.53 ; reverted, 3.67 : insoluble, 0.91 : tota1, I8.II : available, I7.20.

\section{BALTIMORE PULVERIZING CO.,}

\section{Baltinore, Maryland.}

62I. Special Fall Mixture, R. N. Stewart \& Son, Agent, Martinsburg, W. Va. Guarantee: Phosphoric acid, insoluble. I : total, 9: available, 8; potash, I; phosphoric acid from Flor- 
ida dissolved bone phosphate; potash from sulphate of potash. Found: Phosphoric acid, soluble, 0.92 ; reverted, 7.83 ; insoluble, I.02; total, 9.77 ; available, 8.75 ; potash, 1.75 ; chlorine, 2.6 .

Remark: Source of potash equivalent to kainit.

\section{GRIFFITH \& BOYD COMPANY,}

Baltimore, Maryland.

6roo. IX Potash Manure, T. P. Licklider, Martinsburg, W. Va. Guarantec: Phosphoric acid, soluble, 7; reverted, 3 ; insoluble, I; total, II ; available, Io; potash, 5; phosphoric acid from S. C. rock; potash, $1 / 2$ from muriate of potash, $1 / 2$ kainit. Found: Phosphoric acid, soluble, 4.30; reverted, 6.29; insoluble, 2.IO; total, I2.69; available, I0.59; potash, 4.83: chlorine, ז.6.

6r42. IX Potash Manure, R. Hunter, Agent, Berkeley Springs, W. Va. Guarantee: (As above). Found: Phosphoric acid, soluble, 4.58 ; reverted, 7.20 ; insoluble, 2.39; total, I4.I7; arailable, II.78; potash, 4.82 ; chlorine, I.6.

6r33. High Grade Acid Phosphate, R. Hunter. Agent, Berkeley Springs, W. Va. Guarantee: Phosphoric acid, soluble, I2; reverted, 2; insoluble, I ; total, I5; available, I4; phosphoric acid from S. C. rock. Found: Phosphoric acid, soluble, I3.4I : reverted, 3.35 : insoluble, 0.74: total. I7.50; available, 16.76 .

6r40. Valley Fertilizer, R. Hunter, Agent, Berkeley Springs, W. Va. Guarantee: Phosphoric acid, soluble, 6 reverted, 2: insoluble, I: total, 9: available, 8; nitrogen, 0.4I : potash, 3: phosphoric acid, I-Io from fish tankage, 9-Io from rock: nitrogen, $1 / 2$ from garbage tankage: $1 / 2$ fish: potash, $1 / 3$ 
from muriate of potash, $2 / 3$ from kainit. Found: Phosphoric acid, soluble, 6.50; reverted, 2.39; insoluble, o.86; total, 9.75; available, 8.99 ; nitrogen, 0.51 ; potash, 2.92 ; availability of nitrogen, 69; chlorine, 2.7.

Remark: Source of potash equivalent to kainit.

\section{THE HUBBARD FERTILIZER COMPANY,}

\section{Baltimore, Maryland.}

6r82. Hubbard's Soluble Bone Phosphate, submitted for analysis by M. W. Burr, Bardman, W. Va. Guarantee: Phosphoric acid, soluble, II; reverted, 5; insoluble, I; total, I7; available, I6; phosphoric acid from high grade Florida phosphate. Found: Phosphoric acid, soluble, 15.66; reverted, 0.58 ; insoluble, 0.24 ; total, I6.48; available, I6.24.

6I83. Hubbard's Dissolved Animal Bone \& Potash, Parley DeBerry, Agent, Terra Alta, W. Va. Guarantee: Phosphoric acid, soluble, 5; reverted, 3; insoluble, I; total, 9; available, 8 ; nitrogen, I; potash, 2 ; phosphoric acid, $3 / 5$ from high grade Florida phosphate, $1 / 5$ from tankage, $1 / 5$ from dissolved animal bone; nitrogen, $1 / 2$ bone tankage; $1 / 2$ blood and animal matter; potash, $1 / 2$ from sylvanit, $1 / 2$ from kainit. Found: Phosphoric acid, soluble, 6.52; reverted, I.74: insoluble, 1.83; total, I0.09; available, 8.26 ; nitrogen, I.06 potash, 2.65 ; availability of nitrogen, 86; chlorine, 2.4 .

6216. Hubbard's Soluble Bone Phosphate, G. T. Hodges, Shepherdstown, W. Va. Guarantee: Phosphoric acid, soluble, II ; reverted, 5 ; insoluble, I ; total, I7 ; available, I6; phosphoric acid from high grade Florida phosphate. Found: Phosphoric acid, soluble, I 5.25; reverted, 2.52; insoluble, 0.84; total, I8.6I; available, I7.77. 
6217. Hubbard's Alkaline, G. 'T. Horges, Agent, Slicpherdstown, $\mathrm{W}^{\top}$. Va. Guarantec: Phosphoric acid, soluble, 7.50 ; reverted, 4.50 ; insoluble, I: total, I3; available, I2 : potash, 3; phosphoric acid from high srade Filorida pliosphate; potash from sylvanit. Found: Phosphoric acid. snluble, 6.73; reverted, 5.IO; insoluble, 0.99; total, I 3.82: available, I2.83: potash, 5.42; chlorine, I.3.

6232. Hubbard's Columbia Gem Phosphate, C. E. Casler, Agent, Berkeley Springs, IV. Va. Guarantee: Phosphoric acicl, soluble, 5 ; reverted, 3 ; insoluble, I : total. 9: available. 8 ; nitrogen, 0.4I; potash, I; phosphoric acid, 3/5 from high srarle Florida phosphate, $I-5$ from tankage, $I-5$ from dissolved animal bone; nitrogen. $\mathrm{T} / 2$ from bone tankage, $\mathrm{T} / 2$ from blood and animal matter; potash, $1 / 2$ from sylvanit. $1 / 2$ from kainit. Found: Phosphoric acid, soluble, $2.8 \mathrm{I}$; reverted, 6.68 : insoluble, 2.88: tota1, I2.37: available, 9.49: nitrogen, 0.89 : potash, I.94, availability of nitrogen, 80 ; chlorine, 2.6.

6233. Hubbard's Oriental Phosphate, G. T. Hodges, Agent, Shepherdstown, W. Va. Guarantee: Phosphoric acid. soluble, 5; ;reverted, 3; insoluble, I; total, 9 ; available. 8; nitrogen, I; potash, I; phosphoric acid, $3 / 5$ from high grade Florida phosphate, I-5 from tankage, I-5 from dissolved animal bone; nitrogen, $1 / 2$ from bone tankage, $1 / 2$ blood and animal matter; potash, $1 / 2$ from sylvanit, $1 / 2$ from kainit. Found: Phosphoric acid, soluble, 8.2I ; reverted, 2.25 ; insoluble, I.45; total, Ir.9I ; available, I0.46; nitrogen, 0.83 : potash, I.86; availability of nitrogen, 82 ; chlorine, 2.6 .

Remark: Nitrogen low. 


\section{6 \\ T'HE JARECKI CHEMICAL COMPANY}

Station P., Cincinnati, Ohio.

6029. No. I Fish Guano, Harbour \& Burdett, Agent, IIurricane, IV. Va. Guarantee: Phosphoric acid, soluble, 6; reverted, 2; insoluble, I; total, 9; available, 8; nitrogen, I; potash, 2; phosphoric acid from mineral phosphate. bone and animal tankage; nitrogen from animal tankage; potash from muriate: Found: Phosphoric acid, soluble, 5.77; reverted, 2.48 ; insoluble, I.65; total, 9.90 ; available, 8.25 ; nitrogen, I.I3; potash, 2.24; availability of nitrogen, 7I; chlorine, I.3.

Remark: Chlorine excessive.

603I. No. I. Fish Guano, The Damron Feed \& Seed Co.. Agent, Huntington, W. Va. Guarantee: (As above). Founcl: Phosphoric acid, soluble, 5.68; reverted, 3.37 ; insoluble, т.80; total, I0.85; available, 9.05 ; nitrogen, I.I2; potash, 2.27 ; availability of nitrogen, $7 \mathrm{I}$; chlorine, I.3.

Remark: Chlorine excessive.

6115. No. I Fish Gnano, J. M. Rodgers, Agent, Ronceverte, W. Va. Guarantee: (As above). Found: Phosphoric acid, soluble, 5.37 ; reverted, 3.22 ; insoluble, г.65; total, Io.14: availalbe, 8.49 ; nitrogen, I.I2; potash, I.91 ; availability of nitrogen, 75 ; chloride, r.6.

Remark: Chlorine excessive.

6030. Fish \& Potash, Tobacco \& Potato Food, Harbonr \& Burdett, Agent, Hurricane. W. Va. Guarantee: Phosphoric acid, soluble, 6 ; reverted, 2: insoluble, I; total, 9; available, 8: nitrogen, 0.82; potash, 4; phosphoric acid from mineral phosphate, bone and animal tankage: nitrogen from animal tankage; potash from muriate. Found: Phosphoric acid, soluble, $5.3 \mathrm{I}$; reverted, 2.64 : insoluble, 1.73 ; total, 9.68 : avail- 
able, 7.95 ; nitrogen, o.yo; potasli, f.7 : arailability of nitroanen, 73 ; clilorine, 1.4 .

Remark: Chlorine equivalent to mannte salts.

THE MARIETTA BONE \& PHOSPHATE COMPANY, Marietta, Ohio.

6r.49. "Horse Brand" l'otato \& Truck Special, C. li. Braumlick \& Co., Agent, Wheeling, W. Ta. Guaranter: Phosphoric acid, total, 10; available, 8 ; nitrogen, 2.50 : prowi, i) plosphoric acid, $1 / 4$ from tankage, $3 / 4$ from acid phosplane. nitrogen, I/2 from bone tankage, $1 / 2$ from nitrate of solla; petash from muriate. Found: Phosphoric aciel, soluble, 2.35: reverted, 7.51 ; insoluble, 2.14 ; total, 12 ; avalable, y.86; 11 logen, 1.34; potash, 4.93 ; arailability of nitrogen. By: cluk:rine, .9.

Remark: Nitrogen low.

6ı56. "Horse Brand" Potato \& Truck Special, IValter Marshall, Agent, Wheeling, IV. Ta. Guarantee: (As abore). Found: Phosphoric acid, soluble, 3.97; reverted, 5.92; insoluble, I.o4; total, 10.93 ; available, 9.89 ; nitrogen. I.32 : potash, 3.t4; availability of nitrogen, 90: chlorine, .9.

Remark: Nitrogen low. Potash low.

6155. "Horse Brand" Raw Bone, Walter Marshall, Igent. Wheeling. TV. Va. Guarantee: Phosphoric acid, total, 2n: nitrogen, t; phosphoric acid from raw bone: nitrogen from raw bone. Fonnd: Plosphoric acid, total, 21.2.t; nitsowe11, 3.30: availability of nitrogen, 79.

Remark: Nitrogen low.

6r43. "Horse Brand" Acid Phosphate, C. F. Braumlick, Agent, Wheeling, W. Va. Guarantee: Phosphoric acid, total, 


\section{8}

12; available, 10; phosphoric acid from acid phosphate. Found: Hhosphoric acid, soluble, 6.73 ; reverted, 3.95 ; insoluble, 0.45 ; total, 11.13: available, ro.68.

\section{B. MARTIN COMPANY,}

\section{Philadelphia, Norfolk \& Richmond.}

6r94. Martin's Pure Raw Bone Meal, "Bull Head," Washington, Alexander \& Cooke, Charlestown, MV. Va. Guarantee: Phosphoric acid, total, 22 ; nitrogen, 3.7 r pinosphoric ceid from pure raw bone; nitrogen from pure raw brac. Fonnd: I'hosphoric acid, total, 2r.70; nitrogen, 4.03, availability of nitrogen, 79 .

Remark: Phosphoric acid low.

\section{THE NEW PROCESS FERTILIZER COMPANY.}

\section{Columbus, Ohio.}

605I. Humus Chief, M. 'T. 'Thurston, Agent, Charleston, IV. Va. Guarantee: Phosphoric acid, total, I5; available, 1.: phosphoric acid from phosphate rock and tankage. Found: Thosploric acid, soluble, 8.34; reverted, 5.44; insoluble, 2.03 ; total, I 5.81 ; available, I3.78.

6052. O. K. Chief, M. T. 'Thurston, Agent, Charleston. II. Va. Cinarantee: Phosphoric acid, total, 8 ; available, 7 : nitrogen, 1.35 ; potash, 2 ; phosphoric acid from phosphate rock and tankage, nitrogen from garbage tankage, potash from muriate. Found: Phosphoric acid, soluble, 4.43: reverted, 3.63: 111soluble, г.16; total, 9.22; available, 8.06; nitrogen, т.6г ; potash, 2.51 ; availahility of nitrogen, 65 ; chlorine, I.2. 
G053. Little Chiei, M. 'l'. 'A'hurstun, Agent, Charleston, W. Va. Cinarantec: l'hosplouric acid, total, 12; alailable, Io; potash, 3; phosphoric acid from phosphate rock and tankage. putash from muriate. livund: L'hosphoric acid, solnble, 6.14; reverted, 3.66 ; insoluble, 1.36 ; total, II.16; available, 9.70; potash, 2.9I; chlorine, 1.5.

Remark: Phosphoric acid low. Chlorine excessive.

\section{G. OBER \& SONS COMPANY,}

Baltimore, Maryland.

6174. Ober's larmer's Mixture, Standard Hardware Co., Agent, Weston, WI. Va. Gnarantee: Phosphoric acid, solub!e, 7 ; reverted, 2; insoluble, I; total, ro; available, 9 ; nitrogen. 0.82 ; potash, 2; phosphoric acid from high grade Florida phosphate, 8-9; bone from tankage and fish, $1-9$; nitrogen from high grade bone and blood tankage, $1 / 2$ to $3 / 4$; fish, $1 / 2$ to $3 / 4$. Potash from sulphate of potash. Found: Phosphoric acid, soluble, 8.90; reverted, 1.66; insoluble, 0.49; total Ir.05; available, 10.56; nitrogen, I.I3; potash, 2.57 ; availability of nitrogen, 90 ; chlorine, 2.4 .

Remark: Source of potash equivalent to manure salts. Chlorine excessive.

6I8r. Ober's High Grade Acid Phosphate, sent in for analysis by M. WT. Burr, Bardman, IV. Va. Guarantee: Phosphoric acid, soluble, I4.50; reverted, r.50: insolinble, r; total, 17: available, 16; phosphoric acid from high grade Floricla phosphate. Found: Phosphoric acid, soluble, I4.53; reverterl, 2.34; insoluble, 0.40 ; total, 17.27 ; available, 16.87.

6213. S. \& S. Special Wheat Compound, R. N. Stewart \& Son, Agent, Martinshurg, W. Va. Guarantee: Phosphoric acir- soluble, 7 : reverted, 2 ; insoluble, 2 ; total, II ; available, 


\section{0}

y; nitrogen, 0.82 ; potash, 2; phosphoric acid from high grade Florida phosphate, 8-9; bone from tankage and fish, 1-9); 1ntrugen from high grade bone and blood tankage, $1 / 2$ to $3 / 4$; lish, $1 / 2$ to $3 / 4$; potash from sulphate of potash. liound: I'husphoric acid, soluble, 6.55 ; reverted, 2.25 ; insoluble, 0.73 ; tutal, 9.53; available, 8.80 ; nitrogen, I.40; potash, I.92; availability of nitrogen, 89; chlorine, I.4.

Remark: Source of potash equivalent to manure salts.

6192. Ober's Dissolved Bone Phosphate, Washington, Alexander \& Cooke, Agent, Charlestown, W. Va. Guarantee: I'hosphoric acid, soluble, II ; reverted, 3 ; insoluble, $\mathrm{x}$; total, 15; available, I4; phosphoric acid from high grade Filoricla 1)hosphate. Found: Phosphoric acid, soluble, I3.64; reverted, 2.7.3; insoluble, 0.22; total, I6.59; available, I6.37.

6211. Ober's Dissolved Bone Phosphate and Potash, R. N. Stewart \& Son, Agent, Martinsburg, W. Va. Guarantee: Phosphoric acid, soluble, 8; reverted, 2; insoluble, I; total, Ir ; available, ro; potash, 2 ; phosphoric acid from high grade Florida phosphate; potash from sulphate of potash. Found: Phosphoric acid, soluble, 7.88; reverted, 3.16; insoluble, 0.96; total, I2; available, II.04; potash, 2.3I ; chlorine, 2.4 .

Remark: Source of potash equivalent to manure salts. (hlorine excessive.

62r2. Ober's Dissolved Animal Bone, .R N. Stewart \& Sron, Agent, Martinsburg, W. Va. Guarantec: Phosphoric acid, soluble, 8 : reverted, 2 ; insoluble, $r$; total. rr : avai'?ble. 10: nitrogen, 2.47: phosphoric acid from bone: nitroge1: from bine. Found: Phosphoric acid, soluble, 7.96: reverterl, 6.6. : insoluble, o.8I; total, r 5.45: availahle, r 4.64: nitrogen, 2.49; availahility of nitrogen, $9 r$. 
PIEDMON'T' MOUN'T' AIRY GUANO COMPANY,

\section{Baltimore, Maryland.}

6054. Piedmont Special Potash Mixture, E. K. Judy, Agent, Lewisburg, IV. Va. Guarantee: Phosphoric acicl, insoluble, I ; total, II ; available, 10 ; potash, 5 ; phosphoric acid from dissolved phosphate rock; potash, I/2 from muriate of potash, I/2 kainit. Found: Phosphoric acid, soluble, \$.21: reverted, I.7I; insoluble, O.I9: total, IO.II; available, 9.92: potash, 5.57 ; chlorine, I.I.

6055. Piedmont Farmer's High Grade Bone \& Potaslı. F. R. Judy, Agent, Lewisburg, $\mathrm{IV}^{\top}$. Ya. Guarantec: Phos1)hosphoric acid, insoluble, 2 ; total, r2 ; a valablc, ro; potas!, 2; phosphuric acid from S. C. rock: potash from kainit. Found: Phosphoric acid, soluble, 7.34: reverted, 2; insoluble, 0.59: total, 9.93 ; available, 9.34 : potash, 3.26 ; chlorine, .9.

Remark: Phosphoric acid low.

624r. Piedmont Farmer's High Grade Bone \& Potash. C. II. Niswander \& Co., Agent, Parkersburg, W. Va. Guarantee: (As above). Found: Phosphoric acid, soluble, 6.75; reverted, 2.53; insoluble, 0.72 ; total. Io; available, 9.28 ; potash, 2.14 ; chlorine, 2.6.

Remark: Phosphoric acid low.

6056. Piedmont Farmer's Favorite, E. R. Judy, Agent, Lewisburg, W. Va. Guarantee: Phosphoric acid, insoluble. 2 ; total, ro: available. 8 ; nitrogen, o.82; potash, 4; phosphoric acid from dissolved phosphate rock and dissolved bone tankage: nitrogen, $1 / 2$ blood and $1 / 2$ bone tankage; potash, $1 / 2$ from muriate of potash. $1 / 2$ kainit. Found: Phosphoric acid, soluble. 6.59; reverted, r.7r ; insoluble, 0.72; tota1, 9.02; available, 8.30: nitrogen, 0.94 ; potash, 5. I I a a valability of nitrogen, 76 ; chlorine, ז.6. 
6r63. Piedmont High Grade S. C. Bone, Bishop \& Barbe, Agent, Jane Lew, W. Va. Guarantee: Phosphoric acid, insoluble, I ; total, I5; available, I4; phosphoric acid from S. C. rock. Found: Phosphoric acid, soluble, I2.I3; reverted, 2.ro; insoluble, o.21 ; total, I4.44; available, I4.23.

6221. Piedmont High Grade S. C. Bone, Bishop \& Barbc, Agent, Jane Lew, W. Va. Guarantee: (As above). Found: Phosphoric acid, soluble, Io.8o; reverted, 2.56 ; insoluble, 0.35 ; total, I3.7 I ; available, I3.36.

Remark: Phosphoric acid low.

6i66. Piedmont Potato Producer, J. A. Mason \& Co., Agent, Mannington, W. Va. Guarantee: Phosphoric acid, insoluble, 2 ; total, 7 ; available, 5; nitrogen, 2.o9; potash, 6; phosphoric acid from dissolved bone and phosphate rock: nitrogen, $\mathrm{I} / 3$ from bone tankage, $1 / 3$ nitrate of soda and $1 / 3$ blood; potash from high grade muriate of potash. Found: Phosphoric acid, soluble, 6.o9; reverted, o.I7; insoluble, 0.4I ; total, 6.67; available, 6.26; nitrogen, 2 ; potash, 5.50 ; availability of nitrogen, 87 ; chlorine, .9.

Remark: Potash low.

6r68. Levering's Harvest Queen, Bishop \& Barbe, Agent, Jane Lew, W. Va. Guarantee: Phosphoric acid, issoluble, 2 ; total, Io; available, 8 ; nitrogen, 0.82 ; potash. 2 ; whosphoric acid from dissolved phosphate rock and dissnlved bnne tankage; nitrogen, $1 / 2$ from blood and $1 / 2$ bone tankage; wotash, $1 / 2$ from muriate of potash, $1 / 2$ kainit. Found: Phosphoric acid, soluble, 7.19; reverted, I.19; insoluble, 0.54; to^.1. 9.02; available, 8.38; nitrogen, r.22; potash, 2.60: availatL.11: $y$ of nitrogen, 73 ; chlorine, I.7.

6r73. General Crop Grower, J. A. Mason \& Co., Agent, Tannington, W. Va. Guarantee: Phosphoric acid, available, 8: nitrogen, 0.82 ; potash, I ; phosphoric acid from bone phcis. phate; nitrogen, $1 / 2$ from blood and $1 / 2$ from bone tankage; potash, $1 / 2$ from muriate of potash, $1 / 2$ kainit. Found: Phos- 
phoric acid, soluble, 6.60 ; reverted, 1.70 ; insoluble, 0.58 ; 1..tal, 8.88; available, 8.30 ; nitrugen, 0.94 ; potash, 2.02 ; avail:tbility of nitrogen, 67 ; chlorine, I.7.

6220. General Crop Grower, Bishop \& Barbe, Ageri, Jane Lew, W: Va. Guarantee: (As above). Found: Phrsphoric acid, soluble, 6.Io; reverted, 3.42 ; insoluble, 0.69 ; tutál. IO.2I ; available, 9.52 ; nitrogen, 0.85 ; potash, I.58; availabili: of 11itrogen, 76 ; chlorine, 2.4 .

Remark: Chlorine excessive.

6222. Pure Raw Bone Meal, G. W. Niswander \& Cu.. Agent, Parkersburg, $W^{\top}$. Va. Guarantee: Phosphoric acirl total, 23; nitrogen, 3.70; phosphoric acid from bone; nitrogeii from raw bone. Found: Phosphoric acid, total, 23.73; nitrigen, 3.80 ; availability of nitrogen, 64 .

6223. Piedmont Pure Raw Bone Mixture, G. W. Niswander \& Co., Agent, Parkersburg, W. Va. Guarantee: Phosphoric acid, insoluble, 4 ; total, I2 ; available, 8; nitrogen, I.O2 ; potash, 2; phosphoric acid from dissolved bone tankage; nitrogen from bone tankage: potash, $1 / 2$ from muriate, $1 / 2$ kainit. Found: Phosphoric acid, soluble, 5.99; reverted, 4.76 ; insoluble, 2.99; total, I3.74; available, I0.75; nitrogen, I.27; potash. 3.3I ; availability of nitrogen, 86 ; chlorine, 3.3 .

Remark: Source of potash equivalent to kainit. Chlorine excessive.

6і6т. Piedmont Economy, Bishop \& Barbe, Agent, Jane Lew, W. Va. Guarantee: Phosphoric acid, available, Io; nitrogen, 0.20 ; potash, 2 ; phosphoric acid from mineral phusphate; nitrogen, $1 / 2$ from bone tankage, $1 / 2$ blood; potash, $1 / 2$ from muriate, $1 / 2$ kainit. Found: Phosphoric acid, soluble. 6.70; reverted, 2.62 ; insoluble, o.20 ; total, 9.52 ; available, 9.32 ; nitrogen, 0.32 ; potash, I.96; availability of nitrogen, 70 ; chlorine, 2.3 .

Remark: Phosphoric acid low. Chlorine excessive. 


\section{RAISIN MONUMENTAL, COMPANY,}

\section{Baltimore, Maryland.}

6028. Special Formulae for Corn \& Buckwheat, A. P. Russell \& Co., Agent, Buckhannon, W. Va. Guarantee: Phosphoric acid, soluble, 4 ; reverted, 2 ; insoluble, I ; total, 7 ; available, 6; potash, 3; phosphoric acid from high grade Charleston, Florida and Tennessee phosphates; potash, $1 / 2$ to $3 / 4$ from high grade muriate of potash, $1 / 4$ to $1 / 2$ genuine German kainit. Found: Phosphoric acid, soluble, 5.45; reverted, 2.88; insoluble, I.4I ; total, 9.74; available, 8.33 ; potash, 3.63 ; chlorine, :.4.

6069. Acid Phosphate, C. W. Mayers \& Son, Agent, Terra Alta, W. Va. Guarantee: Phosphoric acid, soluble, I2; reverted, 2 ; insoluble, I ; total, 15 ; available, I4; phosphoric acid from high grade Charleston, Florida and Tennessee phosphates. Found: Phosphoric acid, soluble, Io.68; reverted, 4.II ; insoluble, I.75; total, I6.54; available, I4.79.

6154. Acid Phosphate, W. H. Bailey Hardware Co., Agent, Morgantown, W. Va. Guarantee: (As above). Found: Phosphoric acid, soluble, Iol72; reverted, 5.39; insoluble, I.52; total, I7.63; available, I6.I I.

6oyo. Dissolved Bone, C. W. Mayer \& Son, Agent, Terra Alta, W. Va. Guarantee: Phosphoric acid, soluble, 8; reverted, 2 ; insoluble, I ; total, I I ; available, ro; nitrogen, I.65: phosphoric acid from high grade Charleston, Florida and Tennessee phosphates; nitrogen, $1 / 4$ to $2 / 3$ dissolved pure animal bone, $1 / 4$ to $1 / 3$ high grade tankage. Found: Phosphoric acid, soluble, 6.75 ; reverted, 5.26 ; insoluble, 3.01 ; total, 15.02 ; available, I2.0I; nitrogen, I.9I ; availability of nitrogen, 88 .

6o7r. Special Bone \& Potash, C. W. Mayer \& Son, Agent, Terra Alta, W. Va. Guarantee: Phosphoric acid, so- 
luble, 8 ; reverted, 2 ; insoluble, I ; total, Ir ; available, Io; potash, 5 ; phosphoric acid from high grade Charleston Florida and Tennessee phosphates; potash, $1 / 2$ to $3 / 4$ from high grade muriate of potash, , $1 / 4$ to $1 / 2$ genuine German kainit. Found: Phosphoric acid, soluble, 4.78; reverted, 5.83 ; insoluble, r.65: total, I2.26; available, Io.6r ; potash, 5.83 ; chlorine, r.4.

6072. Bone \& Potash, C. IV. Mayer \& Son, Agent. Terra Alta, W. Va. Guarantee: Phosphoric acid, soluble, 8; reverted, 2 ; insoluble, I; total, II ; available, Io; potash, 2 ; phosphoric acid from high grade Charleston, Florida and Tennessee phosphates; potash, $1 / 2$ to $3 / 4$ from high grade muriate of potash, $1 / 4$ to $3 / 4$ German kainit. Found: Phosphoric acid. soluble, 6.96; reverted, 3.63; insoluble, I.38; total, II.97: available, 10.59; potash, 2.97 ; chlorine, 1.4.

609r. William Penn Crop Grower, A. P. Russell \& Co.. Agent, Buckhannon, W. Va. Guarantee: Phosphoric acid. soluble, 6; reverted, 2; insoluble, I; total, 9; available, 8: nitrogen, 0.82 ; potash, $\mathrm{x}$; phosphoric acid from high grade Charleston, Florida and Tennessee phosphates; nitrogen, $1 / 3$ to $1 / 4$ from high grade fish, $1 / 3$ to $1 / 2$ high grade tankage, $1 / 6$ to $1 / 5$ blood, $1 / 6$ to $1 / 5$ high grade sulphate of ammonia ; potash. $1 / 2$ to $2 / 3$ from high grade muriate of potash, $1 / 3$ to $1 / 2$ genuine German kainit. Found: Phosphoric acid, soluble, 2.44; reverted, 6.40; insoluble, 4.31 ; total, 13.15 ; available, 8.84 ; nitrogen. 1.09: potash, 1.25 ; availability of nitrogen, 72 ; chlorine, .7 .

6092. Irish Potato Special, A. P. Russell \& Co., Agent. Buckhannon, W. Va. Guarantee: Phosphoric acid, soluble. 5 ; reverted, 2 ; insoluble, I ; total, 8 ; available, 7 ; nitrogen, 3.29; potash, 8; phosphoric acid from high grade Charleston, Florida and Tennessee phosphates; nitrogen, $1 / 4$ to $1 / 2$ from high grade fish, $1 / 4$ to $1 / 2$ high grade tankage, $\pi / 4$ to $1 / 2$ sulphate of ammonia; potash, 9-ro from high grade sulphate of potash. I-Io from muriate of potash. Found: Phosphoric acid, solu- 
ble, 5.8I ; reverted, 2.17 ; insoluble, I.4I ; total, 9.39; available, 7.98 ; nitrogen, 3.57 ; potash, 9.56 ; availability of nitrogen, 86 : chlorine, 0.05 .

6r58. XXX Fertilizer, W. H. Bailey Hardware Co.. Agent, Morgantown, W. Va. Guarantee: Phosphoric acid, soluble, 6; reverted, 2 ; insoluble, I ; total, 9; available, 8 ; nitrogen, I.65; potash, 5; phosphoric acid from high grade Charleston, Florida and Tennessee phosphates; nitrogen, I/s to $\mathrm{I} / 4$ high grade fish, $\mathrm{I} / 3$ to $\mathrm{I} / 2$ high grade tankage; $\mathrm{I} / 6$ to $\mathrm{I}-5$ blood, $I / 6$ to $I-5$ high grade sulphate of ammonia; potash, $I / 2$ to $2 / 3$ high grade muriate of potash, $1 / 3$ to $1 / 2$ genuine German kainit. Found: Phosphoric acid, soluble, 5.42 ; reverted, 3.44: insoluble, 2.30; total, II.I6; available, 8.86; nitrogen, I.58; potash, 5.06 availability of nitrosen, 79 : chlorine, .9.

6I75. Wheat, Corn and Oats Mixture, W. H. Bailey Hardware Co., Agent, Morgantown, W. Va. Guarantee: Phosphoric acid, soluble, 6; reverted, 2 ; insoluble, I; total. 9: arailable, 8; nitrogen, 0.4I ; potash, 2; phosphoric acid from high grade Charleston, Florida and Tennessee phosphates; nitrogen, I-5 to $I / 4$ high grade fish. $I / 3$ to $I / 2$ high grade tankage, $I / 6$ to $I-5$ blood, $I / 6$ to $I-5$ high grade sulphate of ammonia; potash, $1 / 2$ to $2 / 3$ from genuine German kainit, $1 / 3$ to $1 / 2$ high grade muriate of potash. Found: Phosphoric acid, soluble, I.30; reverted, 6.52 ; insoluble, 2.62 ; total, Io.44; available, 7.82 ; nitrogen, 0.52 ; potash, 2.25 ; availability of nitrogen, 70 ; chlorine, 2.4 .

6I84. Best Wheat and Grass Producer, C. W. Mayer \& Sons. Agent, Terra Alta, W. Va. Guarantee: Phosphoric acid, soluble, 6; reverted, 2 ; insoluble, I : total, 9; available. 8: nitrogen, I.23: potash, 3: phosphoric acid from high grade Charlseton, Florida and Tennessee phosphates; nitrogen, $I / 5$ to $I / 4$ from high grade fish. $I / 3$ to $I / 2$ high grade tankage. $I / 6$ to $I / 5$ blood, $1 / 6$ tor -5 high, grade sulphate of ammonia: 
potash, $1 / 2$ to $2 / 3$ from high grade muriate of potash, $1 / 3$ to $5 / 2$ genuine German kaint. Found: Phosphoric acid, soluble. 6.60; reverted, 2.II ; insoluble, 2.46: total, II.I7; available. 8.7 I nitrogen, I.3I ; potash, 3.35; availability of nitrocgn, Sr: chlorine, I.

6185. Seawall Special, Geo. Carskadon, Agent, Keyser, W. Va. Guarantee: Phosphoric acid, soluble, 8; reverted, 2 ; insoluble, I ; total, Ir ; available, ro; phosphoric acid from high grade Charleston, Florida and.Tennessee Phosphates; Found: Phosphoric acid, soluble, 9.06; reverted, 2.48; insolible, 0.84 ; total, 12.38 ; available, I I.54.

6r28. Washington, Alexander \& Cooke's Regular Corn Mixture, Washington, Alexander \& Cook, Agent, Charleston, W. Va. Guarantee: Phosphoric acid, soluble, 8; reverted, 2: insoluble, I; total II; available, ro; potash, 3 ; phosphoric acid from high grade Charleston, Florida and Tennessee phosphates; potash, $\mathrm{r} / 3$ to $\mathrm{x} / 2$ from high grade muriate of potash. $1 / 3$ to $\mathrm{I} / 2$ from genuine German kainit. Found: Phosphoric acid, soluble, I.92; reverted, 8.08; insoluble, 2.56: total, I2.56; available, ro; potash, 3.48 ; chlorine, r.4.

6r88. W. A. \& C's, Special Mixture for Wheat, Washington, Alexander \& Cooke, Agent, Charlestown, W. Va. Guarantee: Phosphoric acid, soluble, 7 ; reverted, 2 ; insoluble, I; total, ro: available, 9 ; nitrogen, o.82; potash 2 ; phosphoric acid from high grade Charleston, Florida and Tennessee phosphates: nitrogen, $I / 5$ to $I / 4$ from high grade fish, $I / 3$ to I/2 from high grade tankage, $1 / 6$ to $1 / 5$ blood, $1 / 6$ to $\mathrm{r}-5$ high grade sulphate of ammonia; potash, $1 / 2$ to $2 / 3$ from high grade muriate of potash, $1 / 3$ to $1 / 2$ genuine German kainit. Found: Phosphoric acid, I0.85; reverted, 3.24 ; insoluble, 1.85 ; total, I5.94: available, I3.09; nitrogen, I.IO; potash, 5.87; availability of nitrogen, 83 ; Chlorine, .9. 
6I89. Washington, Alexander \& Cooke's Special Mixture for Wheat, Washington, Alexander \& Cooke, Agent, Charlestown, W. Va. Guarantee: (As above.) Found: Phosphoric acid, soluble, 7.73; reverted, I.30; insoluble, I.91; total, I0.94; available, 9.03; nitrogen, 0.94 ; potash, 2.271; availability of nitrogen, 80 ; chlorine, I.3.

6I9I. Washington, Alexander \& Cooke, Special Mixture for Wheat, Washington, Alexander \& Cooke, Agent, Charlestown, W. Va. Guarantee: (As above.) Found: Phosphoric acid, soluble, 6.65 ; reverted, I.70; insoluble, 2.19; total, I0.54; available, 8.35 ; nitrogen, 0.98 ; potash, I. 58 : availability of nitrogen, 80 ; chlorine, .2.

Remark: Potash low.

6Igo. Royal Fish Bone and Poatsh, Washington. Alexander \& Cooke, Agent, Charlestown, W. Va. Guarantee: Phosphoric, acid, soluble, 6; reverted, 2; insoluble, I; total, 9; available, 8 ; nitrogen, 1.65; potash, 3 ; phosphoric acid from high grade Charleston, Florida and Tennessee phosphates; nitrogen, $1 / 2$ to $3 / 4$ from high grade fish. $1 / 2$ to $1 / 3$ high grade tankage, $1 / 5$ to $1 / 3$ animal bone, $1 / 2$ to $2 / 3$ high grade muriate of potash, $1 / 3$ to $1 / 2$ genuine German kainit. Found: Phosphoric acid, soluble, 6.83; reverted, I.52; insoluble, I.28: available, 8.35 ; nitrogen, 1.65 : potash, 3.19 : availability of nitrogen, $9 \mathrm{I}$; chlorine, .9.

6196. Soluble Alkaline Bone, Washington, Alexander \& Cooke, Agent, Charlestown, W. Va. Guarantee: Phosphoric acid, soluble, Io; reverted, 2 ; insoluble, I; total, I3; available, I2; potash, 3; phosphoric acid from high grade Charleston, Florida and Tennessee phosphates; potash, $1 / 2$ to $3 / 4$ from high grade muriate of potash, $1 / 4$ to $1 / 2$ genuine German kainit. Found: Phosphoric acid, soluble, 9.52; reverted, 2.36: insoluble, I.25; total, I3.13; available, II.88; potash, 3.50; chlorine, I.5. 
6r97. Raisin's Half and Half Mixture, Geo. Carskadon, Agent, Keyser, W. Va. Guarantee: Phosphoric acid, soluble, 4; reverted, 2 ; insoluble, I ; total, 7 ; available, 6; potash, 3 ; phosphoric acid from high grade Charleston, Florida and Tennessee phosphates; potash, $1 / 2$ to $3 / 4$ from high grade muriate of potash, $1 / 4$ to $1 / 2$ genuine German kainit. Found: Phosphoric acid, soluble 3.89 ; reverted, 2.80 ; insoluble, 1.28 ; total, 7.97 ; available, 6.69 ; potash, 3.26 ; chlorine, 1.6.

6r99. Genuine German Kainit, Washington, Alexander \& Cooke, Agent, Charlestown, W. Va. Guarantee: Potash, I2 ; potash from kainit. Found: Potsh, I3.60; chlorine, 2.6.

\section{SWIFT \& COMPANY,}

\section{Chicago, Illinois.}

6ro4. Swift's Pure Bone Meal, Geo. Carskadon, Agent. Keyser, W. Va. Guarantee: Phosphoric acid, total, 25 ; nitrogen, 2.50; phosphoric acid from bone; nitrogen from bone. Found: Phosphoric acid, total, 26.51; nitrogen, 2.37; availability of nitrogen, 90 .

Remark: Nitrogen low.

6242. Swift's Pure Bone Meal, Geo. Carskadon, Agent, Keyser, W. Va. Guarantee: (As above.) Found: Phosphoric acid, total, 26.38; nitrogen, 2.72; availability of nitrogen, 90.

\section{SWIF'T'S FERTILIZER WORKS.}

Atlanta, Ga., Wilmington, N.C.

6r95. Swift's Superphosphate Standard Grade Guano, Washington, Alexander \& Cooke, Agent, Charlestown, W. Va. Guarantce: Phosphoric acid, soluble, 5; reverted, 3; insoluble, I.50; total, 9.50 : available, 8 ; nitrogen, I.65; potash, 
2; phosphoric acid from bone phosphate of lime and animal bone; nitrogen from No. I ground tankage, meat blood and bone; potash from muriate of potash. Found: Phosphoric acid, soluble, 6.96; reverted, 2.33 ; insoluble, 0.42 ; total, 9.7 I ; available, 9.29; nitrogen, I.74; potash, I.82; availability of nitrogen, 93 ; chlorine, 2.3 .

Remark: Source of potash equivalent to manure salts.

\section{SOUTHERN FERTILIZING COMPANY.}

$$
\text { York, Pa. }
$$

6234. Farmer's Mixture, W. C. Hovemale \& C. A. Rider, Agents, Berkeley Springs, W. Va. Guarantee: Phosphoric acid, soluble, 8 ; reverted, 2 ; insoluble, I ; total, II ; available, ro; potash, 2; phosphoric, acid from dissolved phosphate rock, potash, from kainit. Found: Phosphoric acid, soluble, 6.I4; reverted, 4.47 ; insoluble, 0.56; total, II.I7; available, Iо.6I ; potash, $2.5 \mathrm{I}$; chlorine, 3.6.

Remark: Chlorine excessive.

6235. Royal Wheat and Grass Grower, W. C. Hovemale, and C. A. Rider, Agents, Berkeley Springs, W. Va. Guarantee: Phosphoric acid, soluble, 6; reverted, 2 : insoluble, I; total, 9; available, 8; nitrogen, o.4I : potash, I ; phosphoric acid from dissolved animal bone and dissolved phosphate rock; nitrosen from animal tankage: potash from kainit. Found: Phosphoric acid, soluble, 5.65 ; reverted, 3.92 ; insoluble. o.86; total, I0.43; available, 9.57 ; nitrogen, 0.38 : potash, I.90; availability of nitrogen, 70 ; chlorine, 4.5 .

Remark: Chlorine excessive. 
TUSCARORA FERTILIZER COMPANY.

Baltimore, Maryland.

6rzo. Acid Phosphate, R. Hunter, Agent, Berleciey Springs, W. Va. Guarantee: Yhosphoric acid, soluble, I2; reverted, 2 ; insoluble, 2 ; total, 16 ; available, 14 ; phosphoric. acid from acid phosphate. Found: Phosphoric acid, soluble, II.58; rererted, 3.I4; insoluble, O.I5; total, I4.87; available, I 4.72 .

6г3г. Ammoniated Phosphate, R. Hunter, Agent, Berkeley Springs, W. Va. Guarantee: Phosphoric acid, soluble, 5: reverted, 2 ; insoluble, 1 ; total, 8 ; available, 7 ; nitrogen, 0.82 ; potash, I; phosphoric acid from animal bone and acid phosphate: nitrogen, $1 / 5$ to $3 / 5$ from bone, $2 / 5$ to $4 / 5$ from bone tankage; potash from kainit. Found: Phosphoric acid, soluble, 5.22: reverted, 2.26; insoluble, 0.63; total, 8.II: available, 7.48 ; nitrogen, o.70; potash. I.24; availability of nitrogen, 79; chlorine, 3.I.

Remark: Nitrogen low. Chlorine excessive.

6r39. Farmer's Mixture, .R Hunter, Agent, Berkeley Springs, W. Va. Guarantee: Phosphoric acid, soluble, 4; rererted, 2 ; insoluble, 1 ; total, 7 ; available, 6 ; potash, 3 ; phosphosric acid from acid phosphate: potash, $1 / 3$ to $2 / 3$ from kainit. $1 / 3$ to $2 / 3$ from muriate. Found: Phosphoric acid, soluble, 5.40; reverted, 2.32 ; insoluble, 0.54 ; total, 8.26 ; available, 7.72 ; potash, 2.90 ; chlorine, 3.4 .

Remark: Chlorine excessive.

\section{A. THOMAS \& COMPANY,}

Hagerstown, Maryland.

6I 52. Golden Sheaf, Sent in for analysis by J. H. Sparrow, Hedgesville, W. Va. Guarantee: Phosphoric acid solu- 
ble, 6 ; reverted, 2 ; insoluble, I ; total, 9 ; available, 8 ; nitrogen, 0.8235 ; potash, I; phosphoric acid from high grade acid phosphate; nitrogen $f$ rom garbage tankage; potash from kainit. Found: Phosphoric acid, soluble, 3.33; reverted, 4.87 ; insoluble, 1.23; total, 9.43; available, 8.20 ; nitrogen. 1.08; potash, I.I I a vailability of nitrogen, 87 ; chlorine, 3.3 .

Remark: Chlorine excessive.

6204. Soluble Bone and Potash, Ira Hauck. Agent, Falling Waters, W. Va. Guarantee: Phosphoric acid, soluble, 8; reverted, 2 ; insoluble, I ; total, II ; available, Io; potash, 2 ; phosphoric acid from high grade phosphate rock; potash from kainit. Found: Phosphoric acid, soluble, 6.58; reverted, 3.60; insoluble, 0.35 ; total, I0.53; available, I0.18: potash, 2.80 ; chlorine, 3.I.

Remark: Chlorine excessive.

6205. Farmer's Wheat and Grass Mixture, Ira Hauck, Agent, Falling Waters. W. Va. Guarantee: Phosphoric acid, soluble, 6; reverted, 2 ; insoluble, I; total, 9; available, 8 ; nitrogen, 0.8235 ; potash, 2 ; phosphoric acid from high grade acid phosphate; nitrogen from garbage tankage; potash from kainit. Found: Phosphoric acid, soluble, 5.42; reverted, 3.27; insoluble, 0.42 ; total, 9.I I ; available, 8.69 ; nitrogen, 0.87 : potash, 2.28; availability of nitrogen, $8 \mathrm{I}$; chlorine, 2.6.

\section{P. THOMAS \& SON COMPANY.}

I0oo Drexel Building, Philadelphia, Pa.

6208. Superior Superphosphate, T. P. Licklider, Agent, Martinsburg, W. Va. Guarantee: Phosphoric acid, soluble, 5; reverted, 2; insoluble, $\mathrm{I}$; total, 8; available, 7 ; nitrogen, 0.80 ; potash, I; phosphoric acid from dissolved phosphate rock and dissolved rough bone tankage: nitrogen, $1 / 2 \%$ from ground fish and bone tankage: 3-10\% from dissolved rough 
tankage; potash from German kanit. Found: Phosphoric acid, soluble, 4.66; reverted, 2.15; insoluble, o.8I ; total, 7.62 : available, 6.8I ; nitrogen, o.80; potash, I.I5; availability of 11itrogen, 75 ; chlorine, 2.7 .

6209. Thomas Wheat and Corn Fertilizer, T. P. Licklider, Agent, Martinsburg, W. Va. Guarantee: Phosphoric acid, soluble, 7 ; reverted, 2 ; insoluble, I ; total, Io; available, 9; nitrogen, o.8o; potash, 3; phosphoric acid from dissolved phosphate rock and dissolved rough bone tankage; nitrogen, $1 / 2 \%$ from ground fish and bone tankage; $3-10 \%$ from dissolved rough tankage; potash from German potash salts, $2 \%$ from kainit, I\% from hard salts (I6\%). Found: Phosphoric acid, soluble, 5.99; reverted, 2.25; insoluble, 0.83; total, 9.07 ; available, 8.24 ; nitrogen, 0.90 ; potash, 2.93 ; availability of $\mathrm{ni}$ trogen, $7 \mathrm{I}$; chlorine, I.7.

Remark: Phosphoric acid low.

\section{VIRGINIA CAROLINA CHEMICAL COMPANY,}

\section{Baltimore, Maryland.}

6058. Virginia Carolina Chemical Co.'s Special Bone and Potash, E. R. Judy, Agent, Lewisburg, W. Va. Guarantee: Phosphoric acid, soluble, 8 ; reverted, 2 ; insoluble, I ; total, I I ; available, Io; potash, 5; phosphoric acid from high grade Charleston, Florida and Tennessee Phosphates; potash, I/2 to $3 / 4$ from high grade muriate of potash, $1 / 4$ to $1 / 2$ genuine German kainit. Found: Phosphoric acid, soluble, 5.46; reverted, 4.68; insoluble, r.09: total, Ir.23; available, ro.I 4 : potash, 4.43 ; chlorine, I.2.

Remark: Potash low.

6r r6. Virginia Carolina Chemical Co., Special Compound for Wheat, E. Shoemaker, Marlinton, W. Va. Guarantee: Phosphoric acid, soluble, 6: reverted, 2 ; insoluble, I; total, 9 ; available, 8 ; nitrogen, 0.82 ; potash, I : phosphoric acid from 


\section{I4}

high grade Charleston, Florida and Tennessee phosphates; nitroge11, I- 5 to $1 / 4$ from high grade fish, $1 / 3$ to $1 / 2$ high grade tankage, $I / 6$ to $1 / 5$ blood, $I / 6$ to $I-5$ high grade sulphate of ammonia; potash, $1 / 2$ to $2 / 3$ from high grade muriate of potash, $1 / 3$ to $1 / 2$ genuine German kainit. Found: Phosphoric acid, soluble, 5.23 ; reverted, 3.04 ; insoluble, 2.26; total, I0.53; available, 8.27 : nitrogen, 0.92 ; potash, I.o6; availability of nitrogen, 82 ; chlorine, .+.

6r37. V. C. C. Co.'s Champion Manure. C. E. Casler, Agent, Berkeley Springs, W. Va. Guarantee: Phosphoric acid, soluble, 6 ; reverted, 2 ; insoluble, $\mathbf{x}$; total, 9 ; available, 8 ; nitrogen, $0.4 \mathrm{I}$; potash, I; phosphoric acid from high grade Charleston, Florida and Tennesee phosphates; nitrogen, I-5 to $I / 4$ from high grade fish, $1 / 3$ to $1 / 2$ high grade tankage, $1 / 6$ to $I / 5$ blood, $I / 6$ to $I-5$ high grade sulphate of ammonia; potash, $1 / 2$ to $2 / 3$ from high grade muriate of potash, $1 / 3$ to $1 / 2$ genuine German kainit. Found: Phosphoric acid, soluble, I.46; insoluble, 2.37: reverted, 8.32: total, I2.I5; available, 9.78 ; nitrogen, 0.58 ; potash, I.28; availability of nitrogen, 68 ; chlorine, I.4.

6215. V. C. C. Co.'s I 4\% Acid Phosphate, G. T. Hodges, Agent, Shepherdstown, W. Va. Guarantee: Phosphoric acid, soluble, I2 ; reverted, 2 ; insoluble, I ; total, I5; available, I4; phosphoric acid from high grade Charleston, Florida and Tennessee phosphates. Found: Phosphoric acid, soluble, I3.53; reverted, 2.80 ; insoluble, o.I9; total, I6.52; available, I6.33.

6ri7. Allison \& Addison Star Brand Guano, E. Shoemaker, Agent, Marlinton, W. Va. Guarantee: Phosphoric acid, soluble, 6 ; reverted, 2 ; insoluble, 2 ; total, Io ; available, 8 ; nitrogen, I. 65 ; potash, I; phosphoric acid, $4 / 5$ from phosphate rock, $1 / 5$ from animal bone; nitrogen, $1 / 4$ from tobacco stems, $1 / 2$ animal tankage, $1 / 4$ fish; potash from sulphate of potash. Tiound: Phosphoric acid, soluble, 5.I8: reverted, 3.97 ; insolu- 
ble, 2.26; total, II.fI : available, 9.I5: nitrogen, I.84: putatsh, I.26; availability of nitrogen, 87 : chlorine, .3 .

61 I9. Allison \& Addison's Little Giant Grain and Grass Grower, J. D. Anderson, Agent, Ronceverte, W. Ta. Guarantee: Phosphoric acid, soluble, 6; reverted, 2 : insoluble, 2 ; total, Io; available, 8 ; nitrogen, 0.82 ; potash, 2 ; phosphoric acid, $4 / 5$ from phosphate rock, $\mathrm{I}-5$ from animal bone: nitrogen, $1 / 4$ from tobacco stems, $I / 2$ from animal tankage, $1 / 4$ from fish ; potash from sulphate of potash. Found: Phosphoric acid, soluble, 3.24; reverted, 5.03; insoluble, I.66; total, 9.93: available, 8.27 ; nitrogen, 0.77 ; potash, r.26; availability of nitrogen, 6I ; chlorine, 2.8 .

Remark: Source of potash equivalent to kainit.

6123. Allison \& Addison B. P. Potash Mixturc, I. M. Miller \& Bro., Agent, Ronceverte, W. Ya. Guarantee: Phosphoric acid, soluble, 7 ; reverted, 3 ; insoluble, I; total, I I : available, ro; potash, 2 ; phosphoric acid from phosphate rock: potash from sulphate of potash. Found: Phosphoric acid, soluble. 5.37; reverted, 5.38: insoluble, I.I6; total, II.9I; available. I0.75: potash, 2.I6; chlorine, I.5.

Remark: Source of potash equivalent to manure salts.

6r 22. S. W. Travers \& Co.'s Dissolved Bone Phosphate, J. M. Miller \& Bro., Agent, Ronceverte, W. Va. Guarantee: Phosphoric acid, soltuble, I I ; reverted, 3 ; insoluble. I : total, I5; available, I4; phosphoric acid from phosphate rock. Found: Phosphoric acid, soluble, 8.66; reverted, 7.14: insoluble, o.6I : total, I6.4I ; available, I 5.80 .

6r 36. Casler's Wheat \& Grass Compound, C. E. Casler, Agent, Berkeley Springs, W. Va. Guarantee: Phosphoric acid, soluble, 4 ; reverted, 2 ; insoluble, $I$; total, $7:$ available. 6 : potash, 3: phosphoric acid from high grade Charleston. Florida and Tennessee phosphates: potash, $1 / 2$ to $3 / 4$ high srade 
muriate of potash, $1 / 4$ to $1 / 2$ genuine German kainit. Found: Phosphoric acid, soluble, 4.30 ; reverted, 4.40 ; insoluble, I.56; total, I0.26; available, 8.70; potash, 3.08 ; chlorine, I.3.

\section{ROBERT A. WOOLDRIDGE COMPANY,}

Baltimore, Maryland.

6075. Liberty Bell Potash Mixture, Geo. A. Rogers, Agent, Rowlesburg, W. Va. Guarantee: Phosphoric acid, soluble, Io; reverted, 2 ; insoluble, I ; available, I2; potash, 3 ; phosphoric acid from dissolved phosphate rock; potash, $1 / 2$ from manure salts, $1 / 2$ muriate of potash. Found: Phosphoric acid, soluble, 7.62 ; reverted, 4.28 ; insoluble, $0.5 \mathrm{I}$; total, I2.4I ; available, II.90; potash, 3.68 ; chlorine, 2.6 .

Remark: Source of potash equivalent to kainit.

6239. Liberty Bell Potash Mixture, Thos. Ferney, Agent. Roanoke, W. Va. Guarantee: (As above). Found: Phosphoric acid, soluble, 5.73 ; reverted, 7.40 ; insoluble, I.59; total, I 4.62 ; available, I 3.03 ; potash, 3.03 : chlorine, 2.2 .

Remark: Source of potash equivalent to manure salts.

6o76. German Potash Mixture, Geo. E. Rodgers, Agent, Rowlesburg, W. Va. Guarantee: Phosphoric acid, soluble., 8; reverted, 2 ; insoluble, I ; available, Io; potash, 2 ; phosphoric acid from dissolved phosphate rock; potash from kainit. Found: Phosphoric acid, soluble, 5.48; reverted, 4.63 ; insoluble, 0.42; total, I0.53 ; available. IO.II: potash, 2.84 : chlorine, 3.9.

Remark: Chlorine excessive.

6077. Triumph Pure Bone Phosphate, Geo. E. Rodgers. Agont. Rowlesburg, W. Va. Guarantee: Phosphoric acid, suluble, 6 ; reverted, 2 ; insoluble, .50 ; available, 8 ; nitrogen, I.23; potash, 4; phosphoric acid. $3 / 4$ from dissolved phosphate 
rock, $1 / 4$ from animal bone and tankage; nitrogen, $2 / 5$ fron fish, $2 / 5$ fron bone tankage, $1 / 5$ from bone; potash, $1 / 2$ from muriate of potash, $\mathrm{J} / 2$ from manure salts Found: Phosphoric acic', soluble, 4.95 ; reverted, 4.02 ; insoluble, I.I5; tola! IO.I: available, 8.97 ; nitrogen, 1.45 ; potash, 4.56 ; availability of 11 itrogen, $8 \mathrm{I}$; chlorine, $\mathbf{\text { I. } 8 .}$

6078. Special Potato and Tobacco Fertilizer, Gen. F. Rudgers, Agent, Rowlesburg, WV. Ia. Guarantee: Phosplioric acid, soluble, 7 ; reverted, 2 ; insoluble, I.50; avaialble, 9; nitrogen, I.64; potash, 5; phosphoric acid, $3 / 4$ from dissolved phosphate rock, $1 / 4$ bone tankage; nitrogen, $2 / 5$ from fish, $3 / 5$ from bone tankage; potash, $1 / 2$ from muriate of potash, $1 / 2$ high grade manure salts. Found: Phosphoric acid, soluble, 5.OI ; reverted, 3.75 insoluble, I.33; total, I0.09; available, 8.76; nitrogen, I.97: potash, 4.79 ; arailability of nitrogen, 82 ; chlorine, I.5:

6237. Special Potato and Tobacco Fertilizer, W. J. Summers, Agent, Little Falls, W. Va. Guarantee: (As above). Found: Phosphoric acid, soluble, 5.22; reverted, $4.3 \mathrm{I}$; insoluble, I. 83 ; total, I . 36 ; available, 9.53 ; nitrogen, I.70: potash, 5.63 ; availability of nitrogen, 92 ; chlorine, I.5.

6079. Florida Acid Phosphate, Geo. E. Rodgers, Agent, Rowlesburg, W. Va. Guarantee: Phosphoric acid, soluble, I2: reverted, 2 ; insoluble, I.50; available, I4; phosphoric acid from dissolved phosphate rock. Found: Phosphoric acid, soluble, I0.45; reverted, 5.I I ; insoluble, 0.97; total, I6.53: available, I 5.56 .

6r67. Chieftain Bone Stock Phosphate, Thos. Ferney, Agent, Roanoke, W. Va. Guarantee: Phosphoric acid, solu, ble, 6 ; reverted, 2 ; insoluble, I.50; available, 8 ; nitrogen, r.64; potash, 2 ; phosphoric acid, $3 / 4$ from dissolved phosphate rock, $1 / 4$ from animal bone and tankage; nitrogen, $2 / 5$ from fish, $3 / 5$ from bone tankage; potash, $1 / 2$ from kainit, $1 / 2$ manure salts. 
Found: Phosphoric acid, soluble, 4.6I; reverted, 4.02; insoluble, I.27; total, 9.90; available, 8.63 ; nitrogen, I.75; potash, 2.04; availability of nitrogen, 86 ; chlorine, 2.5 .

6r78. Old Sledge Phosphate, IV. J. Summers, Agent, Little Falls, W. Va. Guarantee: Phosphoric acid, soluble, Io; reverted, 2 ; insoluble, $\mathrm{r}$; available, I2 ; potash, 5 ; phosphoric acid from dissolved phosphate rock; potash, $2 / 5$ from muriate of potash, $3 / 5$ from manure salts. Found: Phosphoric acid, soluble, 9.16; reverted, 3.27 ; insoluble, 0.72 ; total, I3.15 ; arailable, I2.43; potash, 5.67 ! chlorine, 2.

\section{THE WOOLDRIDGE FERTILIZER COMPANY,}

\section{Baltimore, Maryland.}

6057. Tiger Bone Stock Phosphate, E. R. Judy, Agent, Lewisburg, W. Va. Guarantee: Phosphoric acid, soluble, 5; reverted, 3 ; insoluble, r.5o; total, 9.50; available, 8 ; nitrogen, r.03; potash, 4.50; phosphoric acid, 3/4 from acid phosphate, I/4 from dissolved bone and tankage; nitrogen, $3 / 4$ from animal bone and bone tankage, $1 / 4$ from sulphate of ammonia; potash from muriate of potash. Found: Phosphoric acid, soluble, 4.73 ; reverted, 4.I3; insoluble, I.I8; total, I0.04; available, 8.86; nitrogen, I.I4; potash, 3.73 ; availability of nitrogen, 85 ; chlorine, 2.2 .

Remark: Source of potash equivalent to manure salts.

6ro3. German Alkaline Mixture, Sent in for analysis by R. G. Goodwin, Belington, W. Va. Guarantee: Phosphoric acid, soluble, 7 ; reverted, 3 ; insoluble, I : total, II ; available, Io; potash, 2; phosphoric acid from acid phosphate: potash, $1 / 2$ from manure salts, $1 / 2$ kainit. Found: Phosphoric acid, soluble, 6.43; reverted, 5.6r : insoluble, 0.82; total, г2.86; available, 12.04 : potash, 4.03 ; chlorine, 2. 

